Rowan University

Rowan Digital Works

Theses and Dissertations

6-15-2021

\title{
In situ characterization of fiber-matrix interface debonding via full- field measurements
}

Robert Livingston

Rowan University

Follow this and additional works at: https://rdw.rowan.edu/etd

Part of the Mechanical Engineering Commons

\section{Recommended Citation}

Livingston, Robert, "In situ characterization of fiber-matrix interface debonding via full-field measurements" (2021). Theses and Dissertations. 2915.

https://rdw.rowan.edu/etd/2915

This Thesis is brought to you for free and open access by Rowan Digital Works. It has been accepted for inclusion in Theses and Dissertations by an authorized administrator of Rowan Digital Works. For more information, please contact graduateresearch@rowan.edu. 


\title{
IN SITU CHARACTERIZATION OF FIBER-MATRIX INTERFACE DEBONDING VIA FULL-FIELD MEASUREMENTS
}

\author{
by \\ Robert Livingston
}

\author{
A Thesis \\ Submitted to the \\ Mechanical Engineering \\ College of Engineering \\ In partial fulfillment of the requirement \\ For the degree of \\ Master of Science in Mechanical Engineering \\ at \\ Rowan University \\ May 27, 2021
}

Thesis Chair: Behrad Koohbor, Ph.D.

Committee Members:

Francis M. Haas, Ph.D.

Anu Osta, Ph.D. 
(C) 2021 Robert Anderson Livingston 


\section{Dedications}

This thesis is dedicated to my loving wife and family who always encouraged and supported me, and without which I never would have been able to complete this work.

Also, to my Grandfather who always supported and encouraged me. Despite this, he never got to see me finish my thesis. 


\section{Acknowledgments}

First I would like to acknowledge and extend my deepest gratitude to Dr. Behrad Koohbor, my research advisor, for his guidance and unwavering support throughout the entirety of my Master's research and studies.

I would like to recognize my committee, Dr. Francis M. Haas and Dr. Anu Osta, for their guidance and support throughout my studies. I would also like to acknowledge Dr. Anu Osta for his generosity in allowing me to use the mechanical testing and 3D printing facilities in his lab.

I would like to express my sincere gratitude to my fellow graduate students: Kazi Zahir Uddin, Nicholas Pagliocca, and Oyindamola Rahman for their friendship and assistance in and outside of the lab. I would also like to extend my deepest gratitude to the Advanced Materials and Manufacturing Institute (AMMI) of Rowan University for purchasing the DIC equipment used.

Last and most importantly, I would also like to thank my beloved wife, Aidan, my ever-supportive parents, Keith and Katherine, my wonderful brother, Schuyler, and my devoted cats, Fenwick, Crosby, Katie, and Barney, for supporting me from the very beginning of this journey, always being there no matter the circumstances, unconditional love, and always believing in me even when I didn't believe in myself. 


\author{
Abstract \\ Robert Livingston \\ IN SITU CHARACTERIZATION OF FIBER-MATRIX INTERFACE DEBONDING \\ VIA FULL-FIELD MEASUREMENTS \\ 2020-2021 \\ Behrad Koohbor, Ph.D. \\ Master of Science in Mechanical Engineering
}

Macroscopic mechanical and failure properties of fiber-reinforced composites depend strongly on the properties of the fiber-matrix interface. For example, transverse cracking behavior and interlaminar shear strength of composites can be highly sensitive to the characteristics of the fiber-matrix interface. Despite its importance, experimental characterization of the mechanical behavior of the fiber-matrix interface under normal loading conditions has been limited. This work reports an experimental approach that uses in situ full-field digital image correlation (DIC) to quantify the mechanical and failure behaviors at the fiber-matrix interface. Single fiber model composite samples are fabricated from a proprietary epoxy embedding a single glass rod (macro fiber). These samples are then tested under transverse tension. DIC is used to measure the deformation and strain fields in the glass rod, epoxy, and their interface vicinity. Initiation and propagation of the fiber/matrix debond are discussed. A similar approach is applied on samples that encompass two glass rods with the objective to explore the specific patterns of debonding at the fiber/matrix interface in terms of relative fiber spacing and orientation. Experimental results are complemented by finite element analyses. The findings of this research indicate that the inter-fiber distance and angle play major roles in the interface debond nucleation and propagation as well as matrix failure response in unidirectional composites subjected to transverse tension. 


\section{Table of Contents}

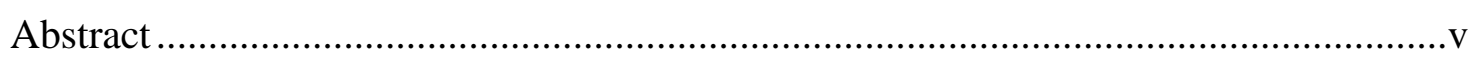

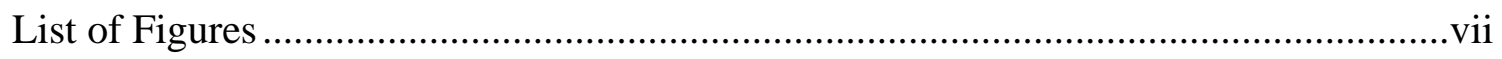

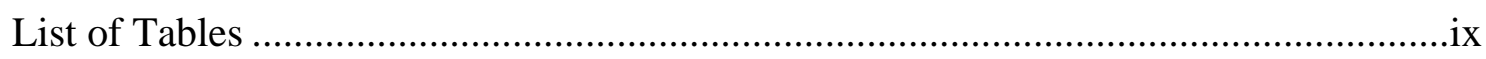

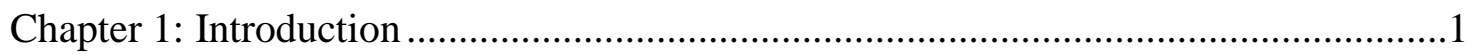

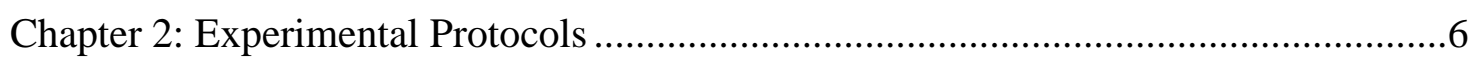

2.1 Materials and Sample Preparation ...................................................................6

2.2 Mechanical Testing and Digital Image Correlation............................................12

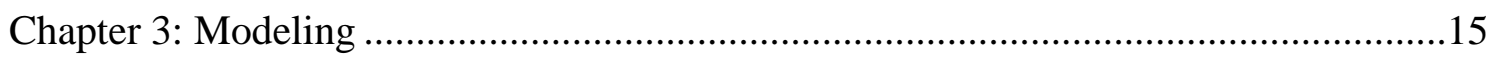

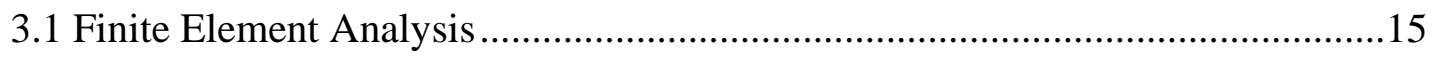

3.2 FEA Mesh and Boundary Conditions …………….........................................15

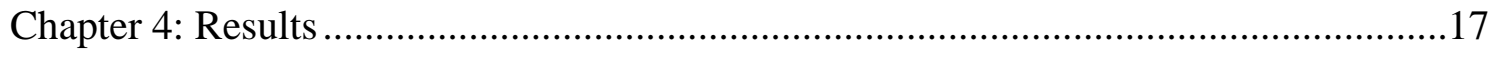

4.1 Single Fiber Samples …………………………........................................17

4.2 Single Fiber Samples: Finite Element Analysis.................................................21

4.3 Identification of Traction-Separation Laws from Single Fiber Tests ...................22

4.4 Double Fiber Samples: Strain Fields ...........................................................2

4.5 Double Fiber Samples: Fiber Interaction .....................................................28

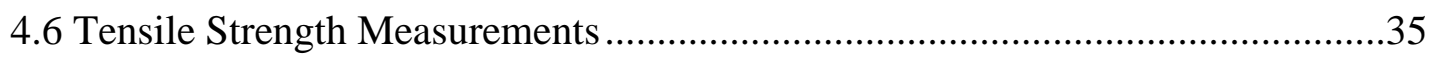

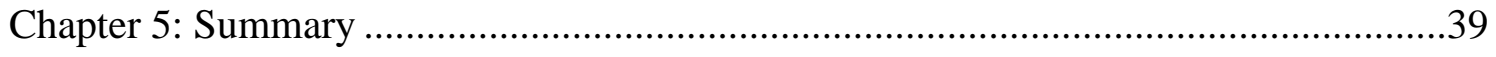

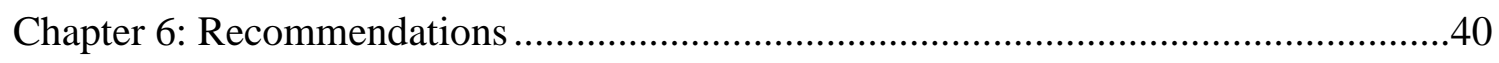

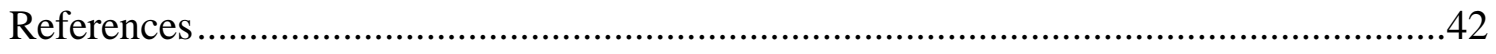




\section{List of Figures}

Figure

Page

Figure 1. Diagram of Debond Propagation and Kinking Out Into The Matrix. Force Applied Is Illustrated by the Arrows. [4].

Figure 2. (a) Stress-Strain Curves Based on Three Independent Tests (b) Correlation Between the Longitudinal (Axial) Strain and Transverse Strain Components Was Utilized to Calculate the Poisson's Ratio of the Neat Matrix Material

Figure 3. 3D Model Representation of Curing Set-Up for Fiber Placement and

Support.....

Figure 4. Schematic Illustrations of (a) Single and Twin Fiber Dog-Bone Samples, and (b) Geometry and Orientation for Location of Fiber Placement. Dimensions of All Samples Were According to ASTM D638

Figure 5. (a) Black Speckle Pattern Used for DIC Applied on the Surface of a SingleFiber Sample. The Large Black Dot Was Used to Make Locating and Tracking of the Glass Macro Fiber Possible. (B) Shows a Close-Up View of the DIC Speckle Pattern. Furthermore, (b) Shows the Physical Size of a Single Subset Used in the DIC Process

Figure 6. (a) Model Geometry and (b) Finite Element Mesh Used for FE Modeling of Single-Fiber Samples. The Displacement Boundary Conditions Used in the Model Were

Extracted Directly From DIC Measurements Are Shown in (c) .16

Figure 7. Evolution of Longitudinal Strain Field $\left(\varepsilon_{y y}\right)$ in the Vicinity of a Single Glass Fiber at Various Global Stresses. Tensile Load Was Applied in Y-Direction

Figure 8. Various Stages of Fiber-Matrix Debond Evolution. Sample Failure Occurred at $41 \mathrm{Mpa}$, in the Form of Complete Separation of the Epoxy Matrix. Dotted Lines on the Lower Right Image Mark the Original Location of the Glass Macro Fiber and the Angular Location of Matrix Crack Nucleation Point .....

Figure 9. (a) Variation of Local Strain Fields Extracted From 4 Representative Locations Around the Fiber With Respect to Global Stress. The 4 Representative Locations Are Selected At the Top, T, Bottom, B, Right, R, and Left, L, of the Fiber. Global Strain Curve Is Plotted for Reference. (b) Evolution of Local Extension/Opening at the Upper and Lowermost Positions at the Interface. Opening Extensions Are Measured Using Virtual Extensometers, $\mathrm{E}_{\mathrm{t}}$ And $\mathrm{E}_{\mathrm{b}}$.... 


\section{List of Figures (Continued)}

Figure

Figure 10. Comparing In-Plane Strain Fields (Normal: $\varepsilon_{y y}, \varepsilon_{x x}$ and Shear: $\varepsilon_{x y}$ ) Developed Around the Glass Macro Fiber in a Single-Fiber Sample Obtained From (A) DIC and (B) FEA. All Contour Maps Are Extracted at a Global Stress of 41 Mpa, i.e., Shortly Before the Sample Failure

Figure 11. (a) Schematic Representation and (b) Actual Traction-Separation Relationships Used to Describe the Cohesive Contact at the Fiber-Matrix Interface. The Scatter Bars Represent the Variability of Experimental Measurements at the Top and Bottom Sides of the Fiber-Matrix Interface.

Figure 12. Evolution of Longitudinal Strain Fields $\left(\varepsilon_{\mathrm{yy}}\right)$ at Various Global Stresses and in

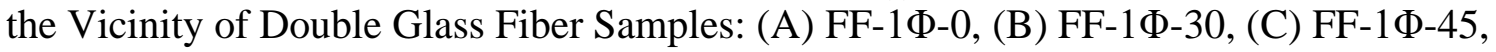

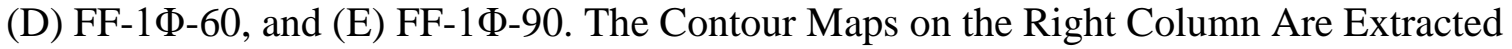
at Global Stresses Just Before Matrix Failure in All Cases. Scale Bar: 5 mm

Figure 13. Evolution of Normal Opening Extension $\left(\Delta \mathrm{L} / \mathrm{L}_{0}\right)$ Extracted From the Upper, $\mathrm{E}_{\mathrm{t}}$, And Lowermost, $\mathrm{E}_{\mathrm{b}}$, Parts of the Fiber-Matrix Interface. For Clarity, Data From the Left Fiber Are Only Provided. Data Shown for (a) FF-1Ф-0, (b) FF-1Ф-30,

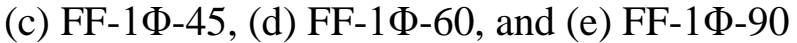

Figure 14. Matrix Failure Angle Data for Double-Fiber Samples With Short (Left Column) And Long (Right Column) Inter-Fiber Distance. Data Shown for Samples With (a) $\mathrm{A}=0^{\circ}$, (b) $\mathrm{A}=30^{\circ}$, (c) $\mathrm{A}=45^{\circ}$, (d) $\mathrm{A}=60^{\circ}$, and (e) $\mathrm{A}=90^{\circ}$. Red Circles Indicate the Original Locations of the Fibers. Scale Bar: $5 \mathrm{~mm}$

Figure 15. (a) Evolution of Local Shear Strain Fields in FF-1 $\phi-30$ at Various Global Stresses. (b) A Magnified View of the Epoxy Ligament Between the Two Fibers, Showing the Location of Matrix Crack Initiation....

Figure 16. (a) Schematic of the Geometric Features of the Shear Band Coalescence Analysis, Sowing the Normal Distance Between the Trajectory of the Shear Bands, Denoted by L. (b) The Correlations Between L, A, and D/ $\phi$ Ratio Shown as by the Color Map. The (A, D/ $\phi$ ) Pairs Wherein the Coalescence of Shear Bands Were Observed (or Not Observed) Are Shown by the Overlayed Black or Hollow Circles

Figure 17. Variation of Tensile Strength for Different Inter-Fiber Distance and Angle Conditions. Tensile Strength of Neat Epoxy and Single-Fiber Samples Are Shown for Comparison 


\section{List of Tables}

Table

Page

Table 1. Mechanical Properties of the Epoxy and Fiber Materials Used in this Work ...8

Table 2. Sample Labels with the Corresponding Nominal and Measured Inter-Fiber

Distance and Angle. .12 


\section{Chapter 1}

\section{Introduction}

Fiber composites are widely used in several areas, from aerospace to automotive industries. Despite their excellent stiffness and load-bearing properties, fiber composites are primarily prone to failure in the form of transverse cracking. Transverse cracking occurs when cross-ply composites are subjected to mechanical loads such as tensile and bending loading conditions. Transverse cracking is initiated when debonding occurs within the fiber-matrix interface. As illustrated in Figure 1, as larger tension loads are introduced the debonded zone propagates around the fiber until it kinks into the matrix creating micrometer cracks. These cracks originate from the debonding area at the fibermatrix interface at the free surface [1]. These cracks coalesce into larger cracks that proliferate throughout the entirety of the transverse lamina [2]. Despite occurring at stresses far less than the ultimate tensile strength of the composite laminate, this cracking is typically the first failure mechanism to occur upon loading of the laminate. After this initial failure, other major failures such as delamination and fiber breakage can occur [3]. 


\section{Figure 1}

Diagram of Debond Propagation and Kinking Out Into The Matrix. Force Applied Is Illustrated by the Arrows [4]
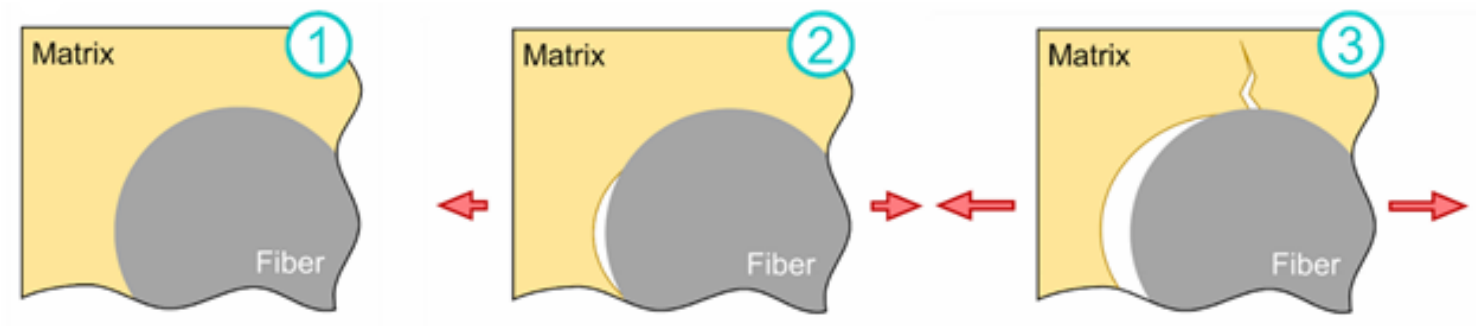

Note. Image reproduced from [4]

Understanding the physics that govern the debonding of the fiber-matrix interface and subsequent transverse crack formation in fiber composites has been at the center of many modeling and analytical methodologies designed for the analysis of composite failure and prevention. The leading areas of research for the past decade have been on finite element analysis (FEA) with a basis in the calculation of energy release rate (ERR) and the cohesive response of the fiber-matrix interface [3,5-11]. While these approaches have been useful in answering some of the fundamental questions, there has been a great lack of experimental verification for these model-based findings. In addition, there still exists a large gap in knowledge concerning the fiber-to-fiber interaction mechanics for microcrack linking, debond crack kinking, and transverse crack formation. One such gap in knowledge is the theory that kinked out microcracks are always linked between the nearest-neighbor fibers [10, 12-14]. Despite generating acceptable results, current experimental evidence fails to support the earlier mentioned assumptions. This lack of experimental evidence is the result of fiber composite transverse cracking having an 
extremely hierarchical and multiscale nature. The fiber-matrix debonding initially occurs at the sub-micron level then propagates to larger cracks that can extend through upwards of several hundred micrometers of lamina. Due to this mechanic, it impedes the creation of experimental techniques capable of capturing the full extent of these failure mechanisms. To compensate, many current experimental techniques, rely on either oversimplified tests where stress/strain states of the test and of the process are no longer consistent with each other, or measurements are taken at length scales that differ by potentially several orders of magnitude from where the failures occur.

3D in situ microtomography has been used for taking measurements of the fibermatrix interface in single fiber samples, and through its use has provided useful quantitative data on the mechanisms and general nature of how damage evolves in the samples [1]. Despite being proven to be exceptionally beneficial for studying the fundamental mechanisms, this microtomography experiment also described being ultimately restricted by the image resolution, prolonged interframe intervals, and the built-in tensile frame's load capacity. In addition, polymer matrix damage due to X-ray beam exposure creates another limiting factor for tomography-assisted experiments $[15,16]$.

Recent innovations in full-field measurements have allowed for increased resolution for in situ measurements during complex loading conditions and over larger length scales (nano to meters). Amongst these innovations, the advancement of digital image correlation (DIC) has proven invaluable for the multiscale characterization of composites $[17,18,19]$. The advent of high magnification DIC has allowed for the multiscale characterization of fiber-matrix interactions and failure mechanisms in 
composites. For example. through the utilization of SEM (scanning electron microscope) DIC it became possible to conduct strain mapping around fibers in both ceramic-matrix [17] and polymer-matrix [4, 20-21] fiber-reinforced composites. Two primary challenges associated with using SEM DIC to analyze transversely loaded fiber-matrix interface are the harmful e-beam charging effects in addition to the irregular nature of image reconstruction in SEM. Alternatively, recently through the use of high magnification optical DIC several studies have investigated interfacial damage mechanisms of transversely loading single-fiber tensile samples [22, 23]. Small-scale stereo DIC now allows for the accurate tracing of interface debonding and crack propagation within the matrix [24]. In all, DIC has become an increasingly more accurate and versatile technique for measuring multiscale displacement and strain fields of fiber-reinforced composites, allowing researchers to further calibrate multiscale finite element models [4].

Numerical models and experimental observations $[1,11]$ suggest that debonding initiates when the energy released during the debonding exceeds the energy dissipated by it. The debond will typically occur perpendicular to the fiber and direction of the applied load at $0^{\circ}$ and $180^{\circ}$ at the free surface. Upon further loading, the debond will grow until reaching about $130^{\circ}$ with respect to the applied load where it will then kink out into the matrix. One study [3] theorized that debonds created during the initial manufacturing process due to either poorly controlled manufacturing processes or stress during the curing process are a likely cause for cracks which then link up, enhancing the debond growth and crack kink-out process.

With the versatility of using DIC to analyze composites, there is a high chance for utilizing its potential to answer questions in how transverse cracking and failure occur. 
This present work seeks to develop an experimental approach to: (1) utilize full-field measurements in the correlation of global stress and strain with local deformation fields around a single fiber in an epoxy matrix, and (2) characterize how local strain fields are affected by fiber spacing and orientation. Through the use of high magnification DIC, we studied the deformation and strain field of the glass rod, epoxy, and their interface are tested under transverse tension to accomplish these goals. Results were then used to characterize the initiation and propagation of the fiber/matrix debond. The data was also used to validate and refine our finite element analysis. A similar approach was then used when testing dual fiber samples to study the effect of spacing and angular orientation of the two fibers. Through these results, the interactions between adjacent fibers were then studied. With these results in conjunction with finite element analyses that were also performed, this work revealed some of the underlying mechanisms that lead to transverse crack formation in unidirectional (UD) composites.

The forthcoming chapters are categorized as follows. In Chapter 2, experimental protocols are discussed. Such protocols include material and sample preparation in addition to the specifications of the parameters for tensile testing and digital image correlation. In Chapter 3, the computational modeling approach is explained. This chapter presents the finite element analysis and a brief comparison with the experimental results. In Chapter 4, the results are presented. These results include those from the experimental tests as well as the complementary FEA results. In Chapter 5, the summary of the work is given. Finally, in Chapter 6, recommendations for future work are presented. 


\section{Chapter 2}

\section{Experimental Protocols}

\subsection{Materials and Sample Preparation}

Dog-bone samples were fabricated from the neat epoxy and based on the ASTM D638 standard. Modulus measurement of the neat epoxy samples (see Figure 2-a) was conducted through optically defined axial deformation in the sample's gauge area. The Poisson's ratio of the epoxy was determined with the in-plane strain fields calculated by DIC. To do this, a correlation was determined between the gauge section's transverse and longitudinal strain components (see Figure 2-b). From this correlation, the Poisson's ratio was determined as the slope of the best linear fit to the in-plane strain data. Due to the data of the blue line in Figure 2-b being questionable the Poisson's ratio was checked with and without its inclusion. With its inclusion the Poisson's ratio was calculated to be 0.40 while without its inclusion the Poisson's ratio was 0.39 . Due to the small difference of only 0.01 the decision to not disregard the possibly errant data was made. This process was repeated for three samples. The neat polymer's mechanical properties were determined to be the averages of the elastic moduli, Poisson's ratios, and failure strains from the three samples. Utilizing the $0.2 \%$ offset technique, $31.7 \mathrm{MPa}$ was established as the yield strength of the material. This yield stress was then utilized to divide the elastic and plastic regions on the stress-strain curve. 


\section{Figure 2}

(a) Stress-Strain Curves Based on Three Independent Tests (b) Correlation Between the Longitudinal (Axial) Strain and Transverse Strain Components Was Utilized to Calculate the Poisson's Ratio of the Neat Matrix Material
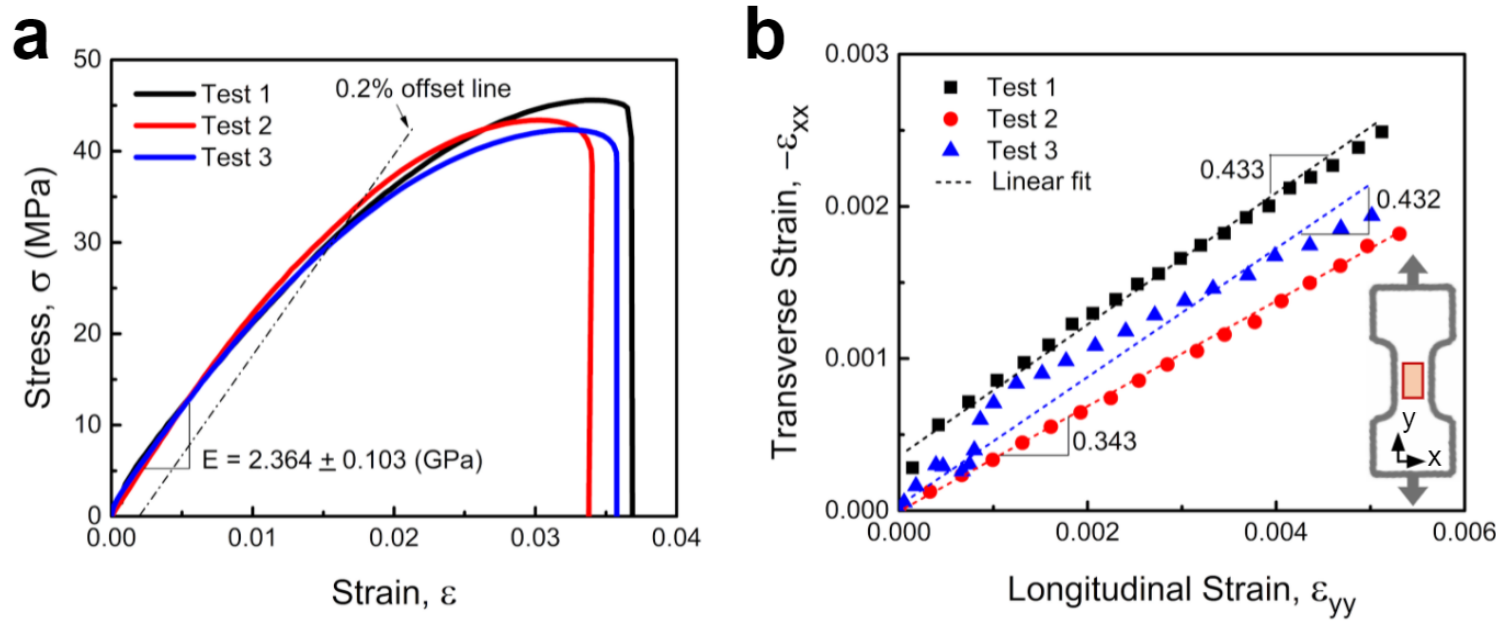

Single fiber samples were prepared by embedding a single glass rod (borosilicate glass, $2 \mathrm{~mm}$ diameter) in a thermoset epoxy resin. The epoxy resin used for the matrix in this work was a proprietary 2-part clear thermoset resin (Naked Fusion Artist Resin) that cures at room temperature. The decision to choose a resin that cures at room temperature was made to minimize the possible undesirable effects on the composite due to a difference in coefficients of thermal expansions (CTE) of fiber and matrix materials. A difference in CTE when fabricating the composite can lead to increased residual stress when using high-temperature curing conditions [25]. Mechanical properties of the glass macro fibers and epoxy matrix are listed in Table 1. 


\section{Table 1}

Mechanical Properties of the Epoxy and Fiber Materials Used in This Work

\begin{tabular}{|c|c|c|c|c|}
\hline Material & $\begin{array}{c}\text { Elastic Modulus } \\
(\mathrm{GPa})\end{array}$ & $\begin{array}{c}\text { Poisson's } \\
\text { Ratio }\end{array}$ & $\begin{array}{c}\text { Tensile Strength } \\
(\mathrm{MPa})\end{array}$ & $\begin{array}{c}\text { Failure Strain, } \\
\varepsilon_{f}\end{array}$ \\
\hline Epoxy $^{*}$ & $2.36 \pm 0.10$ & $0.40 \pm 0.04$ & $43.77 \pm 1.35$ & $0.035 \pm 0.001$ \\
\hline $\begin{array}{c}\text { Glass } \\
\text { Fiber }^{* *}\end{array}$ & 63 & 0.3 & - & - \\
\hline
\end{tabular}

* Based on in-house measurements

** Data provided by the manufacturer

The uncured monomer was first cast into a silicone dog-bone mold in batches of three pairs. A heat gun and needle were then used to eliminate any air bubbles trapped during the mixing process that could cause a weak point in the epoxy matrix. The glass rod was prepared by breaking off a small length from the larger stock then sanding one end until flat with 240 grit sandpaper to ensure the end of the rod is coincident with the surface of the sample and to minimize time spent sanding after curing. To maximize the fiber-matrix interface each glass fiber was cleaned with isopropanol to ensure a clean surface prior to insertion in the resin. After clearing the epoxy of gas bubbles, the rod was inserted perpendicular into the center of each sample in the mold until seated flat at the bottom of the mold. The glass rod was held perpendicular inside the uncured monomer with the aid of a 3D-printed jig (see Figure 3). The custom jig was 3D printed out of ABS with either one (for single fiber samples) or two (for dual fiber samples) holes to facilitate keeping the glass rod correctly positioned during the epoxy curing period. The epoxy was then placed in a fume hood to cure for 24 hours. After such time the samples were removed from the mold and allowed to cure for another 24 hours in a controlled 
environment. Once curing was completed the samples were sanded with 240 then 600 grit sandpaper to remove any imperfections on the surface from the casting and curing phases, ensure a level surface around the testing area of the sample, and ensure the glass fiber is coincident with the surface of the sample.

\section{Figure 3}

3D Model Representation of Curing Set-Up for Fiber Placement and Support

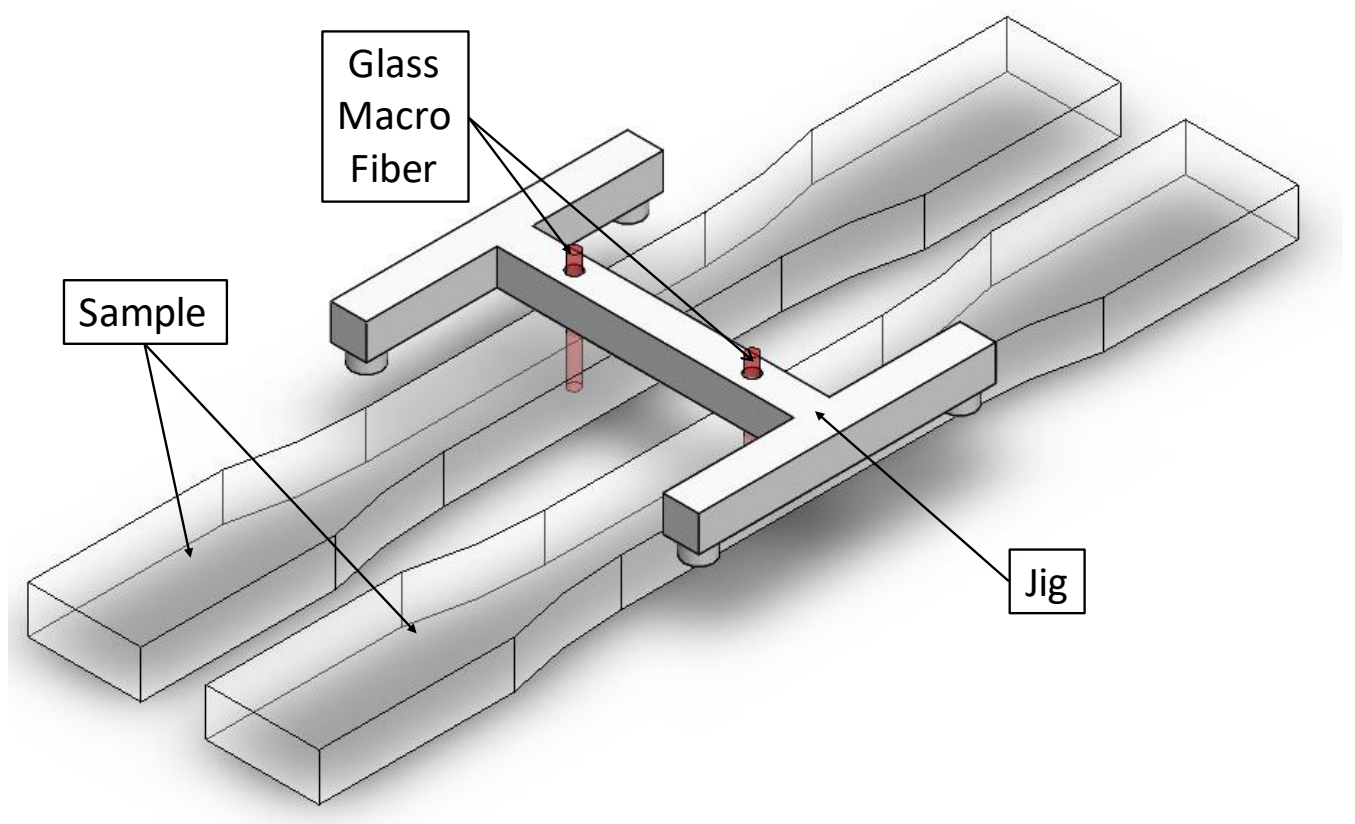

A similar approach was followed to prepare 2-fiber samples. Separate jigs were fabricated and used for each orientation of the two-fiber samples. The jigs were printed on an Ultimaker $2+$ with a $0.6 \mathrm{~mm}$ nozzle with ABS. After printing it was found that the holes as printed were undersized for the glass fiber so then the holes for the glass fiber were brought to a workable size by using a drill bit to slightly enlarge them to a clearance 
fit around the fiber. This level of freedom in the hole was so the jig could be removed easily after casting without any chance of damaging the sample. As illustrated in Figure 4, the spacing between fibers, $d$, and the angular orientation, $\alpha$, between the fibers were varied. The planned samples were to have spacings of $0.5 \phi, 1 \phi$, and $1.5 \phi$ ( $\phi$ indicates fiber diameter). Due to inaccuracies in the jigs due to small scale 3D printing, samples of $0.6 \phi, 2 \phi$, and $3 \phi$ and nominal angular orientations of $0^{\circ}, 30^{\circ}, 45^{\circ}, 60^{\circ}, 90^{\circ}$ with respect to horizontal were prepared and tested. Table 2 lists the nominal and measured values of fiber spacing and angular orientation. Inconsistencies in nominal and measured values were due to slight movement in the fibers during curing and due to minor geometric variations in the $3 \mathrm{D}$ printed jigs. The slight movement was likely due to curing-induced volumetric fluctuations in the epoxy, which were not characterized within this study. A pair of samples for each $(d, \alpha)$ combination were fabricated and tested, to allow for repeatability of experimental results. 


\section{Figure 4}

Schematic Illustrations of (a) Single and Twin Fiber Dog-Bone Samples, and (b) Geometry and Orientation for Location of Fiber Placement. Dimensions of All Samples Were According to ASTM D638

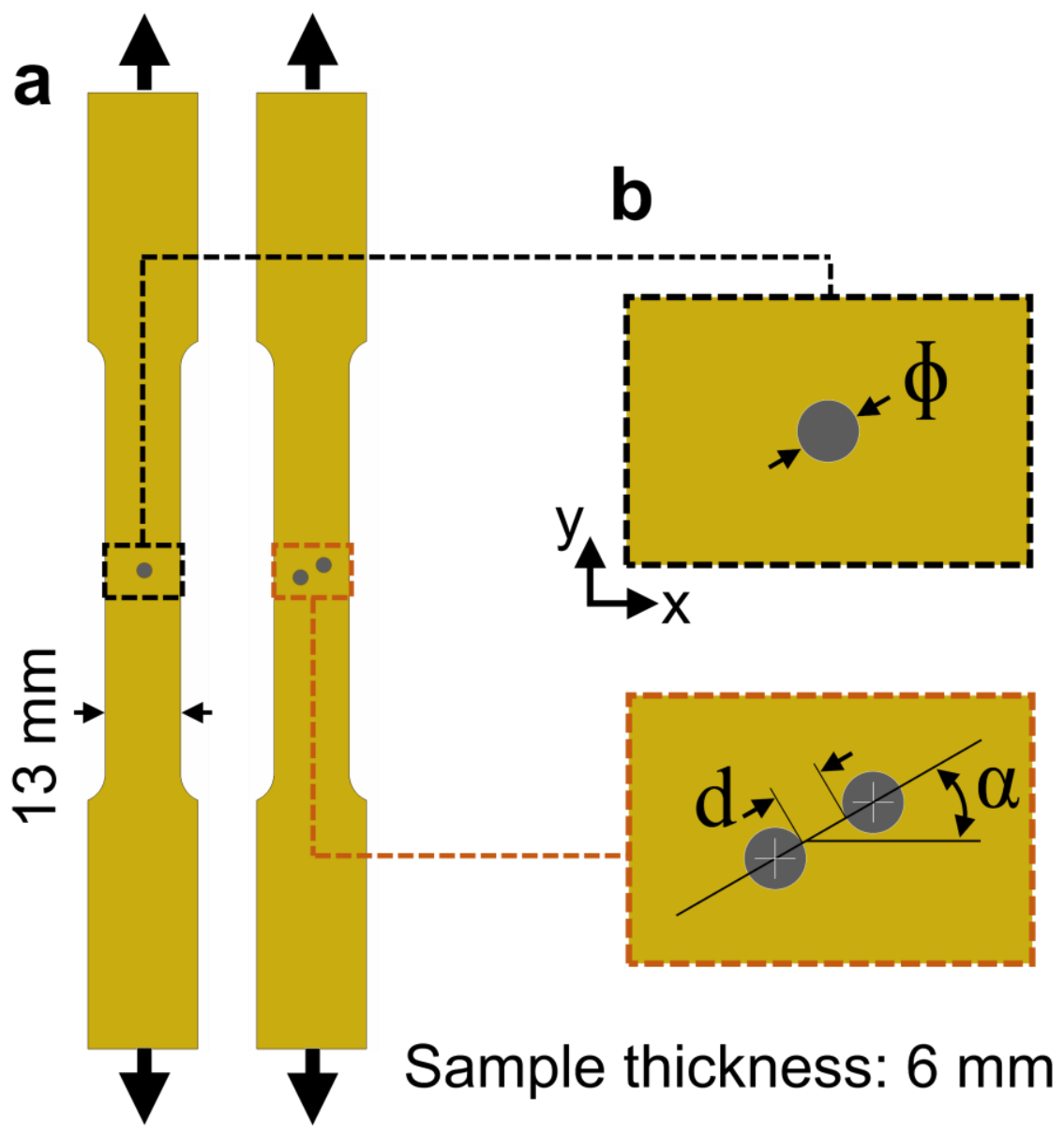




\section{Table 2}

Sample Labels with the Corresponding Nominal and Measured Inter-Fiber Distance and Angle

\begin{tabular}{|c|c|c|c|c|c|}
\hline \multirow{2}{*}{ Sample Label } & \multirow{2}{*}{ No. of fibers } & \multicolumn{2}{|c|}{ Inter-fiber distance, $d(\mathrm{~mm})$} & \multicolumn{2}{|c|}{ Inter-fiber angle, $\alpha\left(^{\circ}\right)$} \\
\hline & & Nominal & Measured & Nominal & Measured \\
\hline $\mathrm{F}$ & 1 & - & - & - & - \\
\hline FF-0.5Ф-0 & \multirow{15}{*}{2} & 0.6 & $0.90 \pm 0.26$ & \multirow{3}{*}{0} & $5.44 \pm 1.34$ \\
\hline FF-1Ф-0 & & 2 & $1.74 \pm 0.24$ & & $4.21 \pm 1.63$ \\
\hline FF-1.5Ф-0 & & 3 & $2.74 \pm 0.11$ & & $0.35 \pm 0.12$ \\
\hline FF-0.5Ф-30 & & 0.6 & $0.71 \pm 0.15$ & \multirow{3}{*}{30} & $38.79 \pm 3.61$ \\
\hline $\mathrm{FF}-1 \Phi-30$ & & 2 & $1.88 \pm 0.23$ & & $32.81 \pm 1.66$ \\
\hline FF-1.5Ф-30 & & 3 & $2.76 \pm 0.05$ & & $31.25 \pm 1.63$ \\
\hline FF-0.5Ф-45 & & 0.6 & $0.52 \pm 0.06$ & \multirow{3}{*}{45} & $46.66 \pm 2.08$ \\
\hline FF-1Ф-45 & & 2 & $1.64 \pm 0.18$ & & $45.97 \pm 0.22$ \\
\hline FF-1.5Ф-45 & & 3 & $2.77 \pm 0.12$ & & $48.53 \pm 1.47$ \\
\hline FF-0.5Ф-60 & & 0.6 & $0.46 \pm 0.06$ & \multirow{3}{*}{60} & $55.14 \pm 4.70$ \\
\hline FF-1Ф-60 & & 2 & $2.09 \pm 0.21$ & & $62.12 \pm 2.07$ \\
\hline FF-1.5Ф-60 & & 3 & $2.77 \pm 0.18$ & & $57.83 \pm 2.19$ \\
\hline FF-0.5Ф-90 & & 0.6 & $0.47 \pm 0.01$ & \multirow{3}{*}{90} & $90.64 \pm 5.49$ \\
\hline FF-1Ф-90 & & 2 & $1.90 \pm 0.12$ & & $88.54 \pm 1.21$ \\
\hline FF-1.5Ф-90 & & 3 & $2.87 \pm 0.09$ & & $90.98 \pm 0.12$ \\
\hline
\end{tabular}

Note. The measured value is the calculated average of two or more samples

\subsection{Mechanical Testing and Digital Image Correlation}

Through the combined utilization of 2D digital image correlation (DIC) and uniaxial tensile testing, characterization of the mechanical behavior of the epoxy as well as the single and double-fiber samples was performed. Following casting, each sample is sanded smooth and then has a pattern applied to its face for DIC testing. To begin, the front face of the samples had a thin initial layer of matte white paint (Rust-oleum ${ }^{\circledR}$ ) applied as a base to enhance the contrast of the DIC speckle pattern. Once the white layer dried a layer of black paint was sprayed directly onto the white surface to create a random 
speckle pattern. This was achieved by suspending the sample horizontally with the white coating facing down. The sample was then speckled by spraying the black paint from a perpendicular direction to the white face for two to three passes then rotating the sample 180 degrees with the white face still facing down and repeating the spray to ensure an even speckle pattern on the white face. This method produced an average speckle particle of $c a .50 \mu \mathrm{m}$. Note that identical speckle application processes were utilized for both the single and double-fiber composite samples. Figure 5 displays an example of a typical speckle patterned sample.

\section{Figure 5}

(a) Black Speckle Pattern Used for DIC Applied on the Surface of a Single-Fiber Sample. The Large Black Dot Was Used to Make Locating and Tracking of the Glass Macro Fiber Possible. (B) Shows a Close-Up View of the DIC Speckle Pattern. Furthermore, (b) Shows the Physical Size of a Single Subset Used in the DIC Process

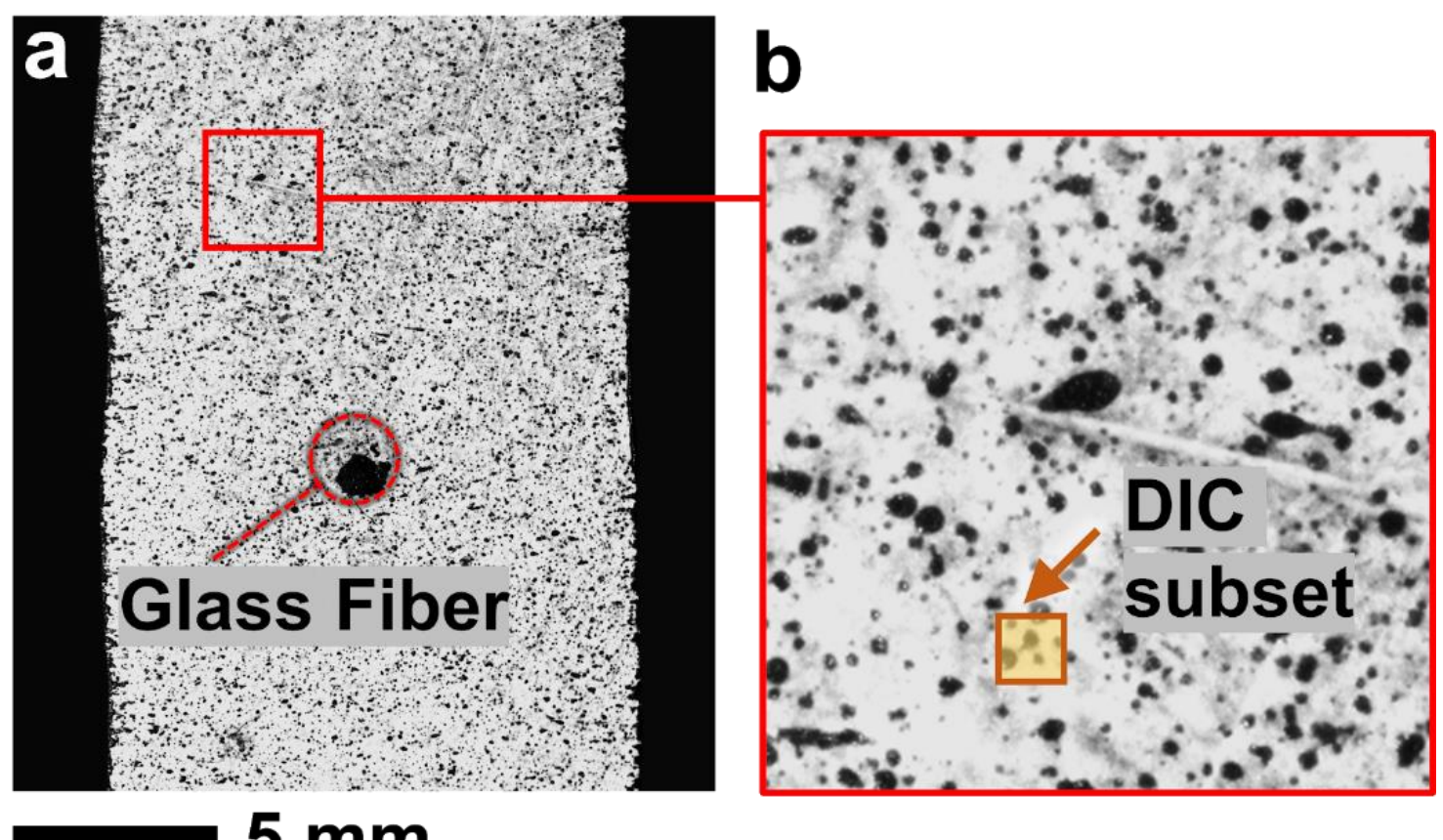


Once the pattern is applied and allowed to dry, the samples were subjected to uniaxial tensile testing until failure occurred. Tensile testing was performed in a Shimadzu $10 \mathrm{kN}$ universal test frame at a consistent crosshead speed of $5 \mathrm{~mm} / \mathrm{min}$. For image acquisition of the speckled surface of the sample, a 5-megapixel camera fitted with a high magnification macroscopic lens was utilized. Data from the load frame and images from the camera were synced to capture data at a rate of $1 \mathrm{~Hz}$. Post-processing of the images captured while testing were analyzed in commercial DIC software (Vic-2D, Correlated Solutions, SC, USA) using a subset of 29 pixels $(272 \mu \mathrm{m})$ and step sizes of 7 pixels $(65 \mu \mathrm{m})$.

To calculate the full-field strain maps, a Gaussian weighted filter size of 5 was used. The parameters applied in the DIC resulted in virtual strain gauge sizes of $c a .330$ $\mu \mathrm{m}$. Due to the small size, this allows for incredibly localized strains to be measured in the vicinity of the fiber-matrix interface. 


\section{Chapter 3}

\section{Modeling}

\subsection{Finite Element Analysis}

To validate and complement the experimental results, a finite element modeling approach was utilized. These finite element models went on to further reinforce the roles that inter-fiber spacing and angle play in the interaction mechanics between fibers, specifically in the two-fiber samples. 2-D models duplicating the geometry of the singlefiber samples were created first. The properties of the individual components, i.e., glass fiber and epoxy matrix were assigned based on the experimentally acquired data, presented earlier in Table 1 and Section 2.2. ANSYS's bonded contact option was used to model the interface between the two components. After verifying the agreement between the FE model results and those of the experiments in the single-fiber case, simulation of the double-fiber sample deformation was performed. In the case of the double-fiber samples, analysis of a wide range of inter-fiber spacing and angles were conducted to study the failure mechanisms and interactions between fibers.

\subsection{FEA Mesh and Boundary Conditions}

Figure 6 illustrates the finite element mesh utilized for the single-fiber FE model as well as the model geometry. The mesh was sufficiently refined to ensure mesh independence. The FE simulation was fixed along the bottom edge in the y-direction. At the same time along the top edge a nonlinear vertical displacement, $\delta$, was applied. The magnitude of this vertical displacement (shown in Figure 6-c) was a direct reflection of the experimental magnitude obtained from the DIC measurements. To model the interface 
contact of the fiber-matrix the traction-separation relationship (as seen in Section 3.3)

was used.

\section{Figure 6}

(a) Model Geometry and (b) Finite Element Mesh Used for FE Modeling of Single-Fiber Samples. The Displacement Boundary Conditions Used in the Model Were Extracted Directly From DIC Measurements Are Shown in (c)

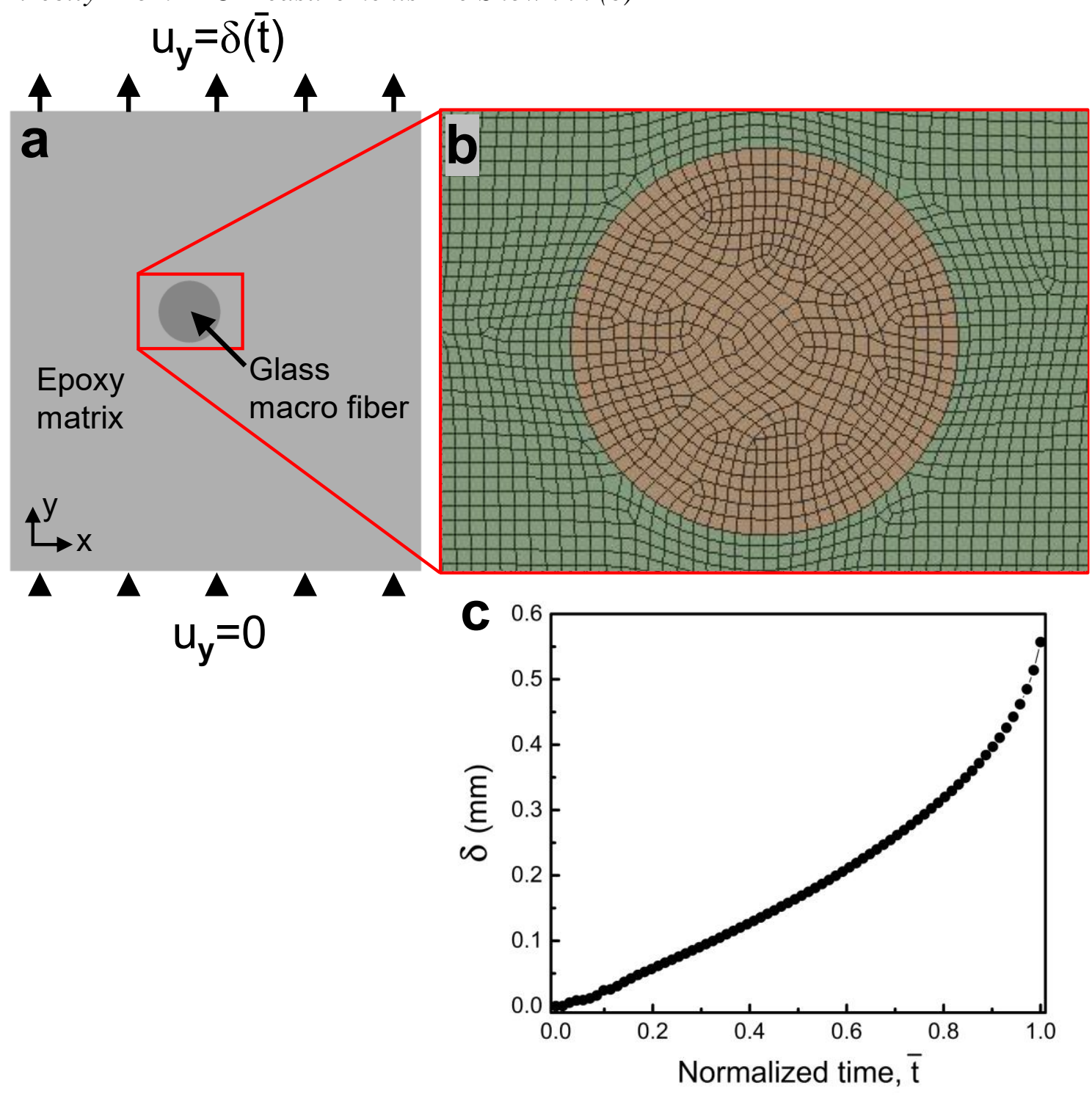




\section{Chapter 4}

\section{Results}

\subsection{Single Fiber Samples}

The development as seen by DIC of the longitudinal strain fields in the singlefiber samples in the area around the glass fiber can be seen in Figure 7. At stresses substantially less than the epoxy's tensile strength, a narrow high strain band is developed in the area surrounding the fiber. As the stress levels around the fiber increase, the highstress band found around the fiber expands. As this occurs the fiber-matrix interface begins to deteriorate until a visible separation between the upper edge of the fiber and matrix can be seen. The genesis of this debonded area develops into an increasingly larger opening along the interface of the fiber and matrix, while simultaneously increasing the strain heterogeneity level in the matrix surrounding the failing fiber-matrix interface. The slight off-center positioning of the fiber within the epoxy dog-bone sample created an obvious asymmetry in the strain maps shown in Figure 7.

\section{Figure 7}

Evolution of Longitudinal Strain Field $\left(\varepsilon_{y y}\right)$ in the Vicinity of a Single Glass Fiber at Various Global Stresses. Tensile Load Was Applied in Y-Direction

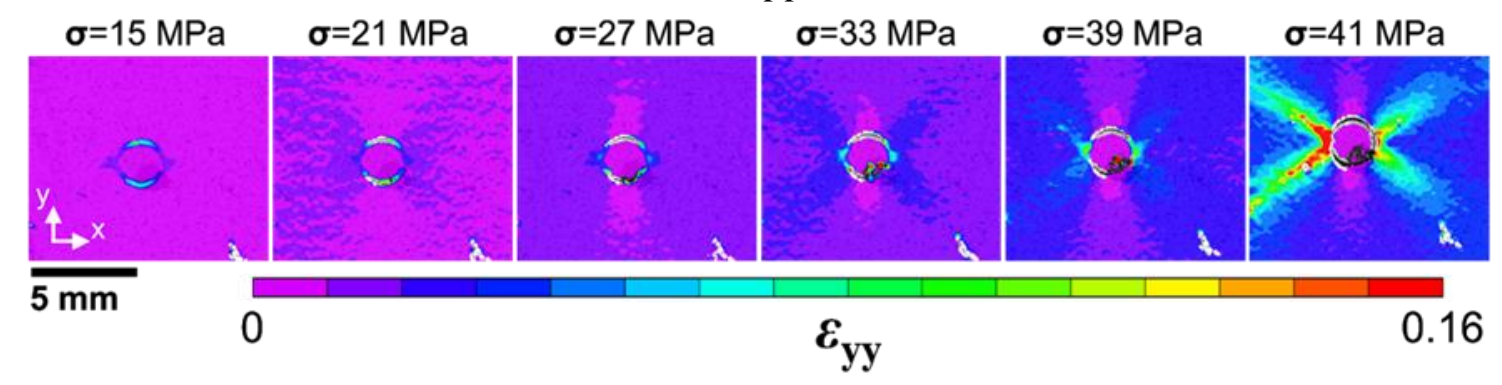


As the global tensile stress rises toward $41 \mathrm{MPa}$, the epoxy suffers a complete failure. Figure 7 illustrates the stages of failure of the single fiber samples as debonding occurred and the propagation characterization. The growth and initiation of the debond in the single fiber samples featured visual characteristics that concurred with the current theory of fiber-matrix interfacial debond $[3,11]$ and also showed significant similarities with observations made at substantially smaller scales for single carbon-fiber samples [4]. The angular location of the crack in the matrix that ultimately resulted in the complete failure of the composite can also be seen in Figure 8. According to previously conducted analytical and modeling research, the nucleation of the matrix crack formation occurs at an angle $<90^{\circ}$ relative to the direction the load is applied. While the experimental observations made during this work agree with those predictions, due to the macro glass fiber being slightly off-center from the center of the sample we are left with uncertainties of whether the oblique-angled location of failure was a direct result of the failure mechanics as stated by the problem, and as predicted by the before mention study, or is a consequence of the less than perfect geometry of the sample tested that ultimately resulted in asymmetry. Despite this, the further quantitative analysis of single fiber local deformation and failure is still possible due to the full-field nature of the strain measurements done in this work. 


\section{Figure 8}

Various Stages of Fiber-Matrix Debond Evolution. Sample Failure Occurred at 41 Mpa, in the Form of Complete Separation of the Epoxy Matrix. Dotted Lines on the Lower Right Image Mark the Original Location of the Glass Macro Fiber and the Angular Location of Matrix Crack Nucleation Point
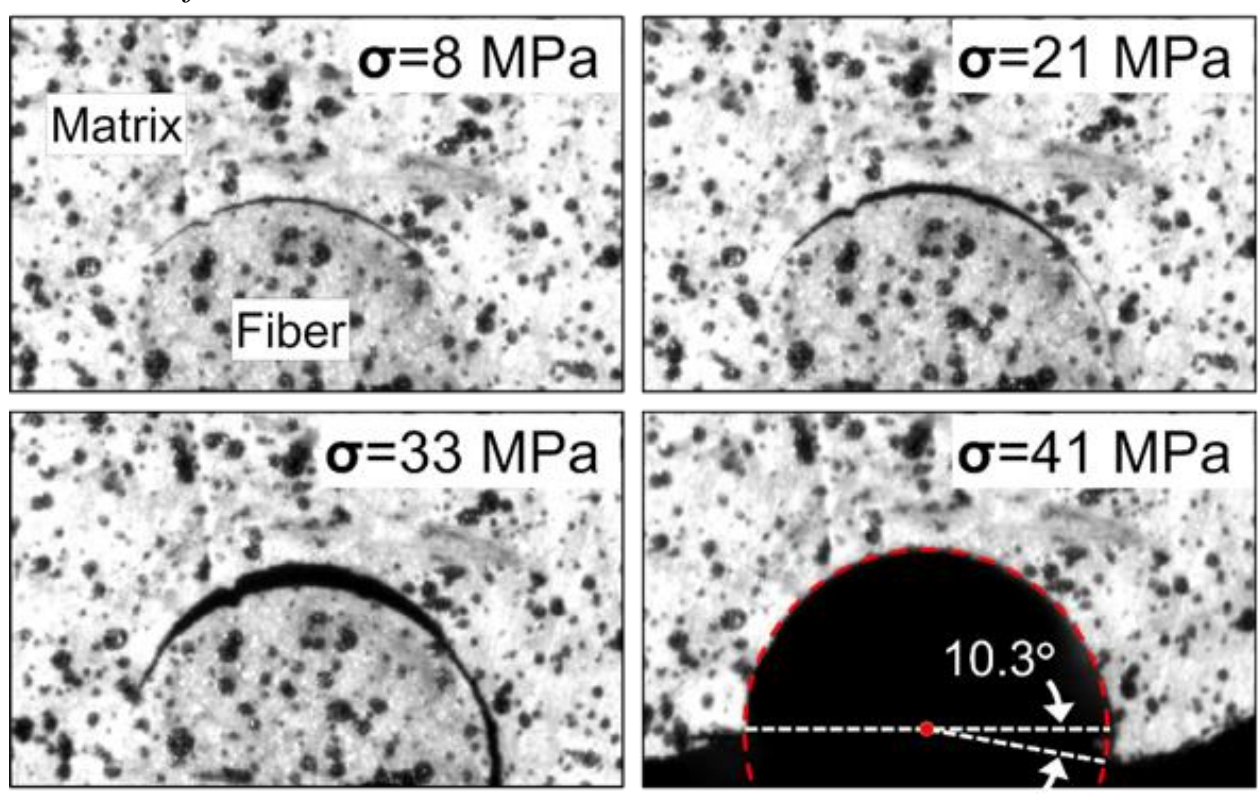

$1 \mathrm{~mm}$

Figure 9 displays the development of local strains and the displacements of the gap between matrix and macro glass fiber as measured at various positions around the fiber and throughout the fiber-matrix interface. Figure 9a illustrates the local strain curves of four representative locations $500 \times 500 \mu \mathrm{m}^{2}$ found $250 \mu \mathrm{m}$ away from the interface. Locations $\mathrm{L}$ and $\mathrm{R}$ feature local strain curves with a distinctly constant increase with increasing stress. In contrast, locations $\mathrm{T}$ and B local strain data display an immediate initial jump of the global stress to a value of $c a .5 \mathrm{MPa}$ (as seen in the Figure 9a inset as the blue arrow mark). After their initial increase, the curves display a discernable dip in value finally leading to a very gradual increase until the failure point is 
reached. The sudden drop in the strain at $c a .5 \mathrm{MPa}$ is the result of the fiber-matrix interface's initial debonding resulting in the matrix partially unloading. This initial debonding of the fiber-matrix interface occurs within the upper and lowermost portions of the interface in tandem at diagonally opposite locations. This pattern of the formation of the debonds is validated by tracing the interfacial gap as explained in Figure 9b.

Another pronounced detail illustrated in Figure 9b, is the sudden increase of the slope of the interface opening curve when the global stress reaches ca. $5 \mathrm{MPa}$. This mirrors the previously mentioned early apex in the strain values in Figure 9a.

\section{Figure 9}

(a) Variation of Local Strain Fields Extracted From 4 Representative Locations Around the Fiber With Respect to Global Stress. The 4 Representative Locations Are Selected At the Top, T, Bottom, B, Right, R, and Left, L, of the Fiber. Global Strain Curve Is Plotted for Reference. (b) Evolution of Local Extension/Opening at the Upper and Lowermost Positions at the Interface. Opening Extensions Are Measured Using Virtual Extensometers, Et And Eb
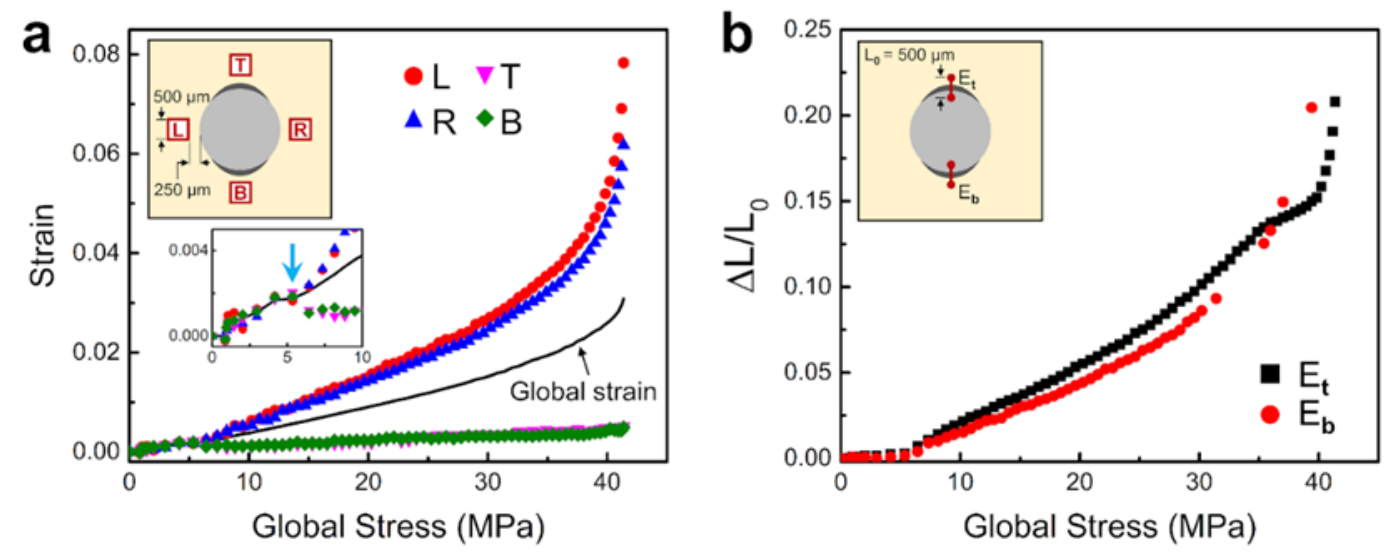

In addition to providing a novel look into the failure mechanics of single fiber samples and their associated multiscale deformations, the measurements displayed in 
Figure 9 were then utilized in the calibration of traction-separation laws (found in Section 3.2) which were necessary for the finite element analysis utilized in this work.

\subsection{Single Fiber Samples: Finite Element Analysis}

Figure 9 displays the strain fields of the single-fiber samples that were acquired through the use of either DIC (Figure 10a) or FEA (Figure 10b) allowing for a direct comparison between them. The contour maps shown in the figure provide a visual representation of both the in-plane normal and shear strain distributions. The finite element model was designed with the glass macro fiber slightly off-center to better replicate the experimental geometric characteristics of the samples tested. After comparing the experimental DIC results and the FEA model predicted results, we found a distinct correlation between them. The modeling results from the FEA closely mirrored the deformation patterns that were characterized in the DIC measurements. The $\varepsilon_{y y}$ and $\varepsilon_{x x}$ maps seen in the figure display highly localized strain bands with orientations measured as $37^{\circ}$ relative to the horizontal of the sample. The locations of maximum shear strain within the interface were then identified as prime nucleation sites for crack kinking. The focus was placed on the angular orientation in addition to the location for the localized strain regions since they are vital pieces of data when characterizing the inter-fiber interactions and patterns of failure in double-fiber samples. 


\section{Figure 10}

Comparing In-Plane Strain Fields (Normal: $\varepsilon_{y y}, \varepsilon_{x x}$ and Shear: $\varepsilon_{x y}$ ) Developed Around the Glass Macro Fiber in a Single-Fiber Sample Obtained From (A) DIC and (B) FEA. All Contour Maps Are Extracted at a Global Stress of 41 Mpa, i.e., Shortly Before the Sample Failure

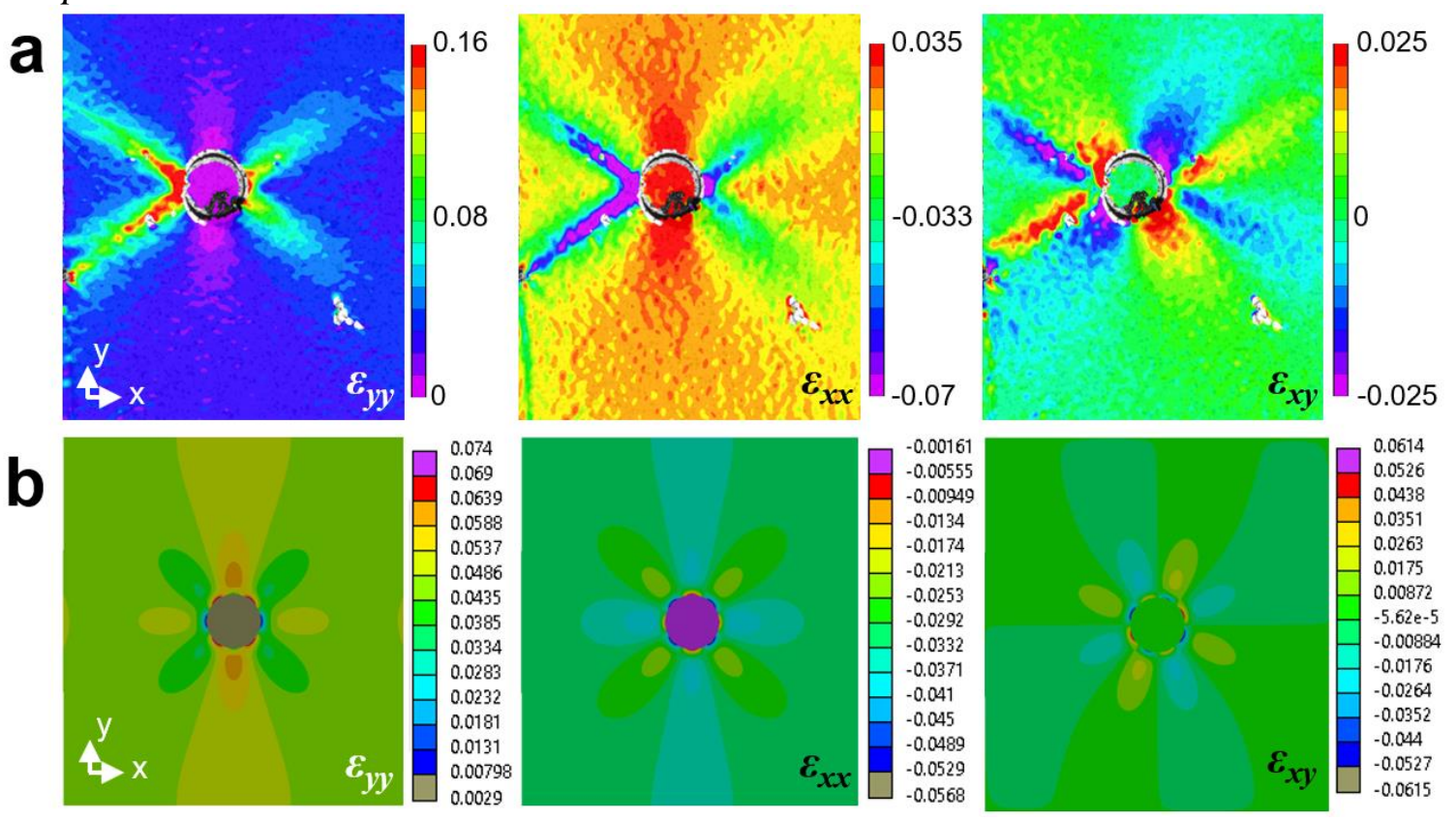

\subsection{Identification of Traction-Separation Laws from Single Fiber Tests}

Cohesive zone traction, $t_{n}(\delta)$, can be theoretically calculated by taking the energy release rate ( $\partial J$ for the cohesive zone [26]) and differentiating it with respect to the displacement of the normal crack opening in the cohesive zone, $\delta_{n}$, i.e.,

$$
t_{n}(\delta)=\frac{\partial J}{\partial \delta_{n}}
$$

Unfortunately, at present in the case of the J-integral at the fiber-matrix interface there does not currently exist an analytical solution [11], as such this approach is not currently viable for use in the present work in identifying traction-separation relationships. 
Nonetheless, due to the work utilizing full-field multiscale deformation measurements we were capable of characterizing the traction-separation relationships directly allowing them to be used for finite element modeling.

As seen in Figure 11a, the fiber-matrix interface cohesive contact behavior was defined for this work as utilizing a triangular traction-separation relationship. This law is described as being defined by three parameters thus why it is known as the triangular traction-separation relationship. The elastic region of the relationship is characterized by taking a linear correlation of the traction (pressure required to form a crack) and the separation. The peak of this region is where damage begins to form as traction forces reach $t_{n}$. This parameter indicates what is effectively the maximum nominal stress possible before damage begins to form. The displacement of the normal opening that corresponds with the traction value where damage initiation occurs is designated as $\delta_{n}^{0}$. The damage evolution region of the graph is defined by a line that links the damage initiation node with $\delta_{n}^{t}$ where the traction reaches zero and complete separation occurs. The slope of this damage evolution line describes the rate at which the damaged zone's load-carrying capacity is reduced. 


\section{Figure 11}

(a) Schematic Representation and (b) Actual Traction-Separation Relationships Used to Describe the Cohesive Contact at the Fiber-Matrix Interface. The Scatter Bars Represent the Variability of Experimental Measurements at the Top and Bottom Sides of the FiberMatrix Interface
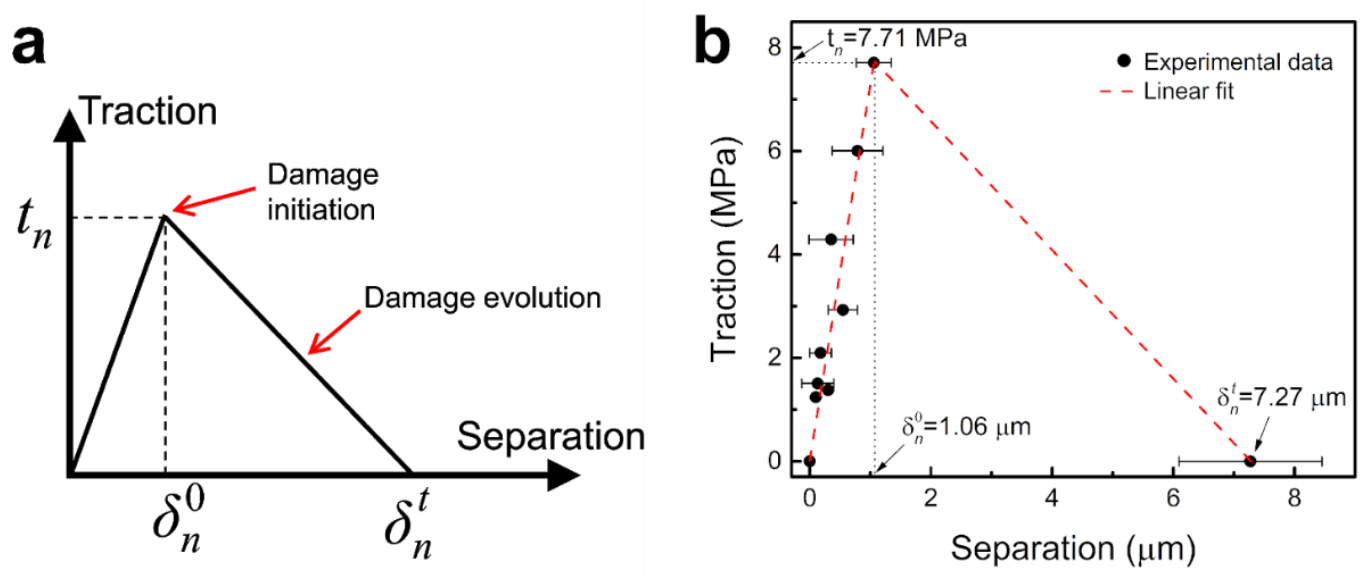

By analyzing our full-field measurements, we can directly extract the three

parameters mentioned. By examining the local strain values obtained for the top and bottom of the fiber-matrix interface (Figure 9a), damage initiation was found to occur at a global tensile stress of $\sigma^{\infty}=5.35 \mathrm{MPa}$. This stress can be evaluated at the interface of the fiber and matrix using the classical solution provided by Goodier [27], and later referenced in [11] as:

$$
\frac{\sigma(\theta)}{\sigma^{\infty}}=k-m \sin ^{2}(\theta)
$$

where, $\theta$ is defined as the polar angle along the interface, $k$ and $m$ are dimensionless elastic bimaterial properties. They are defined by Mantic [28] as functions of Dundurs [29] parameters $\alpha$ and $\beta$ in plane-strain conditions as: 


$$
k(\alpha, \beta)=\left(\frac{1}{2}\right)\left(\frac{1+\alpha}{1+\beta}\right)\left(\frac{2+\alpha-\beta}{1+\alpha-2 \beta}\right), \quad m(\alpha, \beta)=\frac{1+\alpha}{1+\beta}
$$

By utilizing values for the glass/epoxy systems of $\alpha=0.919$ and $\beta=0.229$ [11], the local stresses experienced at the top and bottom-most points of the interface (i.e., $\theta=0^{\circ}$, $180^{\circ}$ ) will yield a value of $t_{n}=7.71 \mathrm{MPa}$. The normal opening displacement associated with $\sigma^{\infty}=5.35 \mathrm{MPa}$ yielded a result of $\delta_{n}^{0}=1.06 \mu \mathrm{m}$ (see Figure 9b). Reusing the previous data set, the global tensile stress in addition to its matching normal opening when total separation occurs were found to be $\sigma^{\infty}=8.82 \mathrm{MPa}$ and $\delta_{n}^{t}=7.27 \mu \mathrm{m}$ respectively.

As demonstrated in Figure 11b, the primary parameters, $t_{n}, \delta_{n}^{0}$, and $\delta_{n}^{t}$, in conjunction with several other required measurement nodes in between are the keys to constructing a functional traction-separation relationship which can also be utilized as an input for finite element modeling. As an additional note, the region beneath the traction-separation curve is indicative of the critical fracture energy of the debonding process which was identified to be $28.03 \mathrm{~N} / \mathrm{m}$.

\subsection{Double Fiber Samples: Strain Fields}

The DIC strain fields for the double-fiber samples can be seen below in Figure 12. This figure displays how the strains for the double fiber samples of the $1 \Phi$ nominal inter-fiber spacing (see Table 2) develop. The results of the experiment presented by the figure show consistency with model predictions given by Sandino et al. [14]. According to previous modeling results that in the case of a double-fiber composite sample, when the angle between the fibers is $\alpha=0^{\circ}$ so that the fibers are located in such a way that they 
are perpendicular to the load direction the results of the failure and debond mechanics will mirror that of the single fiber case. In such a case the effects like debond pattern and deformation field of one of the fibers will have negligible effects on the remaining fiber in the sample. Figure 12a demonstrates this interesting phenomenon wherein the doublefiber sample strain patterns seen developing about each fiber mimic the patterns seen around the single fiber samples (see Figure 7). In samples where $30^{\circ} \leq \alpha \leq 60^{\circ}$ (see Figure 12b-d) the strain fields around the fibers exhibit a distinct interaction within the sample. These interactions manifest as a single localized band of high strain creating a bridge between the fibers. The final case is where $\alpha=90^{\circ}$ thus aligning both fibers parallel to the loading direction (Figure 12e). In this orientation there exists no qualitatively noticeable interaction between the fibers in the sample. 


\section{Figure 12}

Evolution of Longitudinal Strain Fields (cyy) at Various Global Stresses and in the

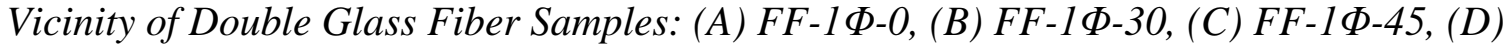

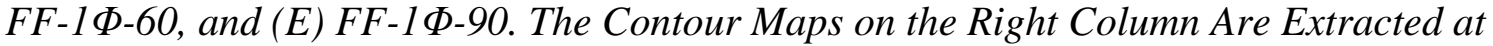
Global Stresses Just Before Matrix Failure in All Cases. Scale Bar: 5 mm
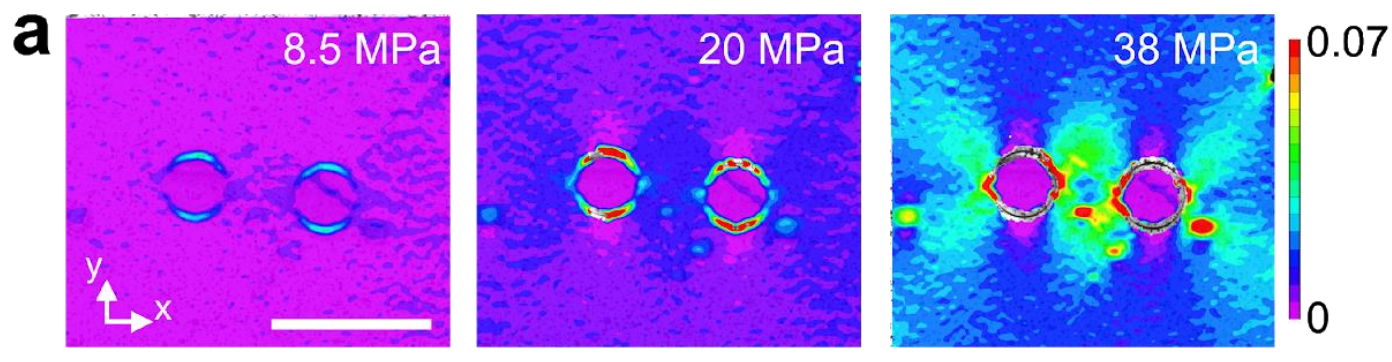

b
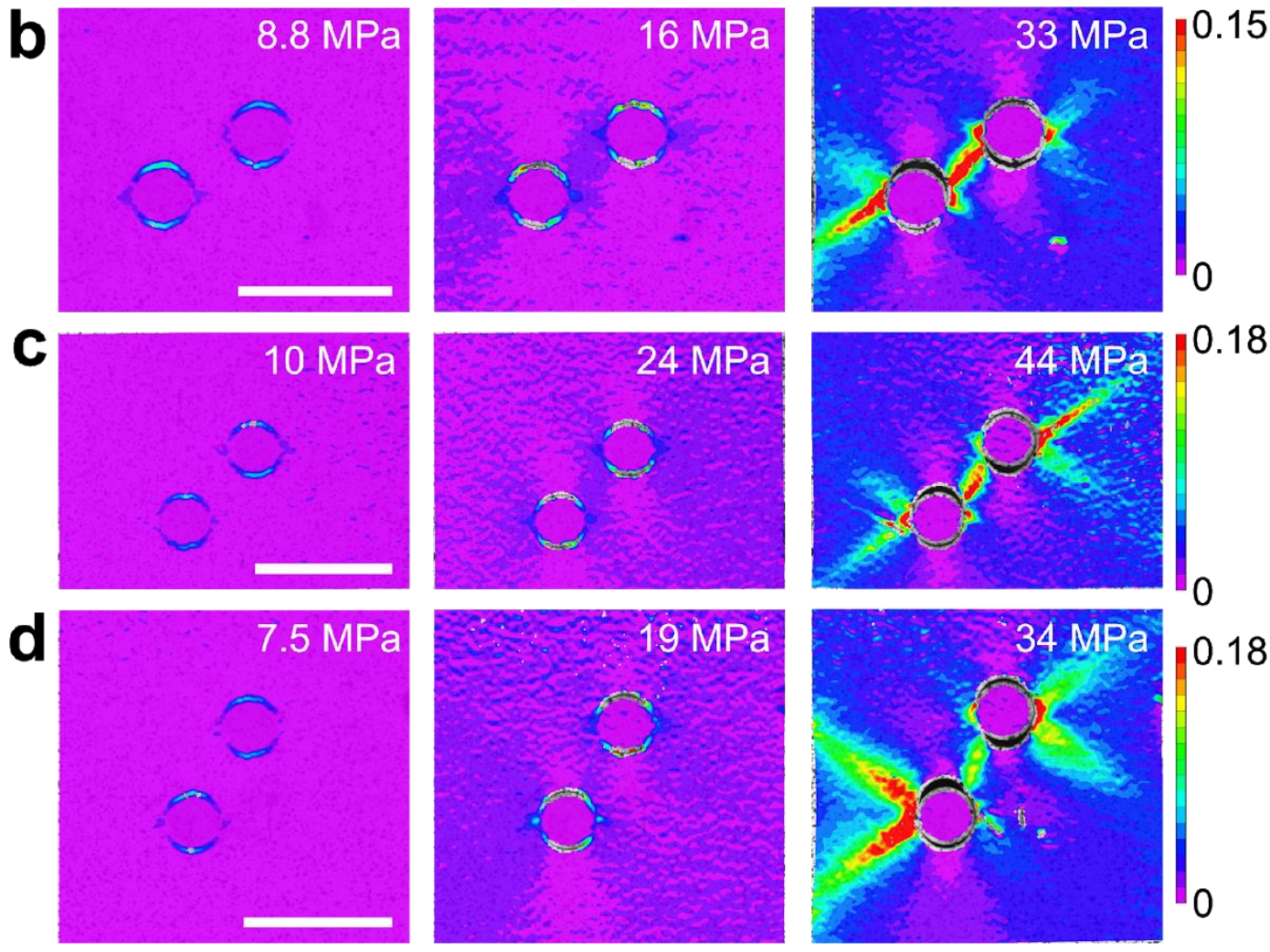

e
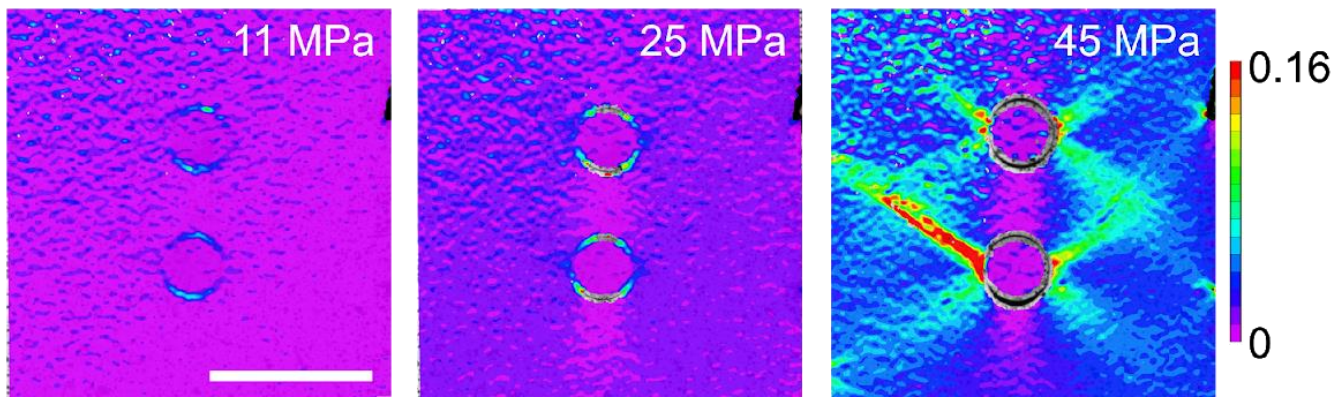


\subsection{Double Fiber Samples: Fiber Interaction}

According to many formerly conducted computational and analytical research, the best way to predict failure in multifiber samples is to utilize a method that relies on analyzing the mechanisms of the fiber interaction through a qualitative assessment by determining the energy release rate. To calculate the energy release rate requires detailed measurements of the sample's local stress fields in addition to the local strain.

Unfortunately measuring the local stress fields experimentally is practically impossible but fortunately, local strain can be acquired from the DIC results directly. Therefore in lieu of linking energy release rate with the failure pattern of the composite, the research utilizes the matrix failure and debonding patterns through the lens of deformation and strain fields generated in the area of surrounding fibers.

To begin the process of analyzing, the symmetry of the debonding in twin-fiber samples is studied. Figure 13 illustrates the progression of the matrix-fiber interface gap displacement in twin-fiber samples at the upper and lowermost locations of each fiber. Note that this figure only provides the data for the samples where the nominal fiber spacing was approximately one fiber diameter. As stated previously when the fibers are arranged in an $\alpha=0^{\circ}$ orientation the opening displacement occurs as a result of a symmetric debond. The quantitative values of the gap displacement in this case nearly mirrored the quantities measured for the single fiber case (Figure 10b). This result was found to be consistent with prior modeling studies where it was found that when the fibers are arranged in such a way that their orientation with each other is perpendicular to the tensile load's direction, $\alpha=0^{\circ}$, it leads to a situation where the effects of neighboring fibers become negligible. Unlike when $\alpha=0^{\circ}$, as $\alpha>0^{\circ}$ the opening displacement becomes 
distinctly asymmetrical. For $\alpha=45^{\circ}$ an intriguing response occurs which is not entirely comprehended. At $\alpha=45^{\circ}$ a symmetric response reaching a peak global stress of $c a$. 40 MPa was observed. Another remarkable characteristic observed was the initial slow growth of the slopes of the opening displacement curves at small global stresses. With the use of insets in Figure $\mathbf{1 3}$ the growth of these slopes can be qualitatively characterized. The only graphs that don't display this initial restricted growth in the rate of displacement are $\alpha=60^{\circ}$ and $90^{\circ}$. Previous modeling studies [14] support these findings due to debond propagation being found to require overall reduced tensile loads to initiate at these higher alpha values, i.e., $\alpha=60^{\circ}$ and $90^{\circ}$. Finally, the inter-fiber angle, $\alpha$, was found to be one of the primary drivers for the maximum opening gap created before the total failure of the matrix occurred. The values of $\alpha=0^{\circ}$ and $90^{\circ}$ were found to yield the smallest opening displacements measured while $\alpha=30^{\circ}$ and $60^{\circ}$ had the largest. When $\alpha=45^{\circ}$ was measured it was found to have an opening displacement before ultimate failure between $\alpha=30^{\circ}$ and $60^{\circ}$ but somewhat closer to that of $\alpha=60^{\circ}$. 


\section{Figure 13}

Evolution of Normal Opening Extension (LL/LO) Extracted From the Upper, Et, And Lowermost, Eb, Parts of the Fiber-Matrix Interface. For Clarity, Data From the Left

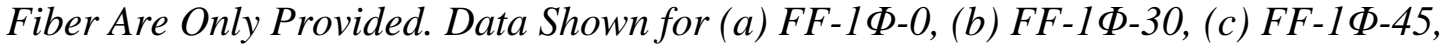

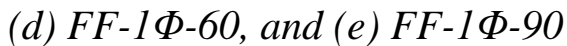

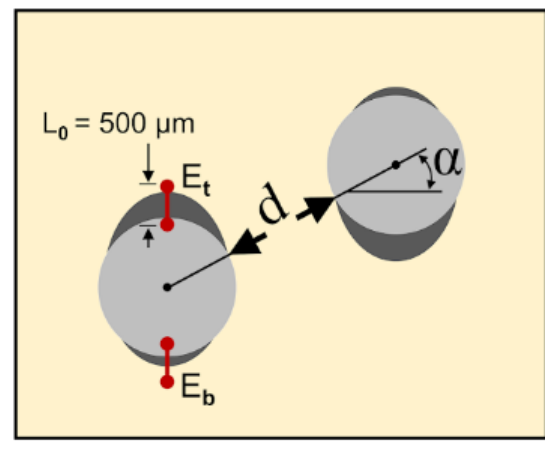

b
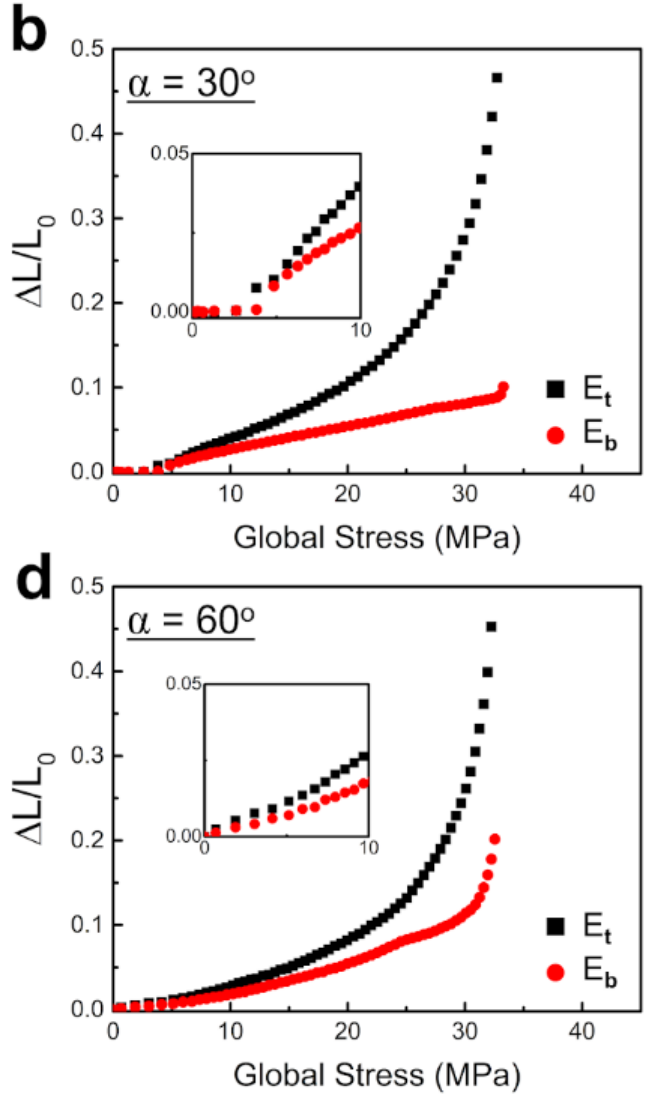

a
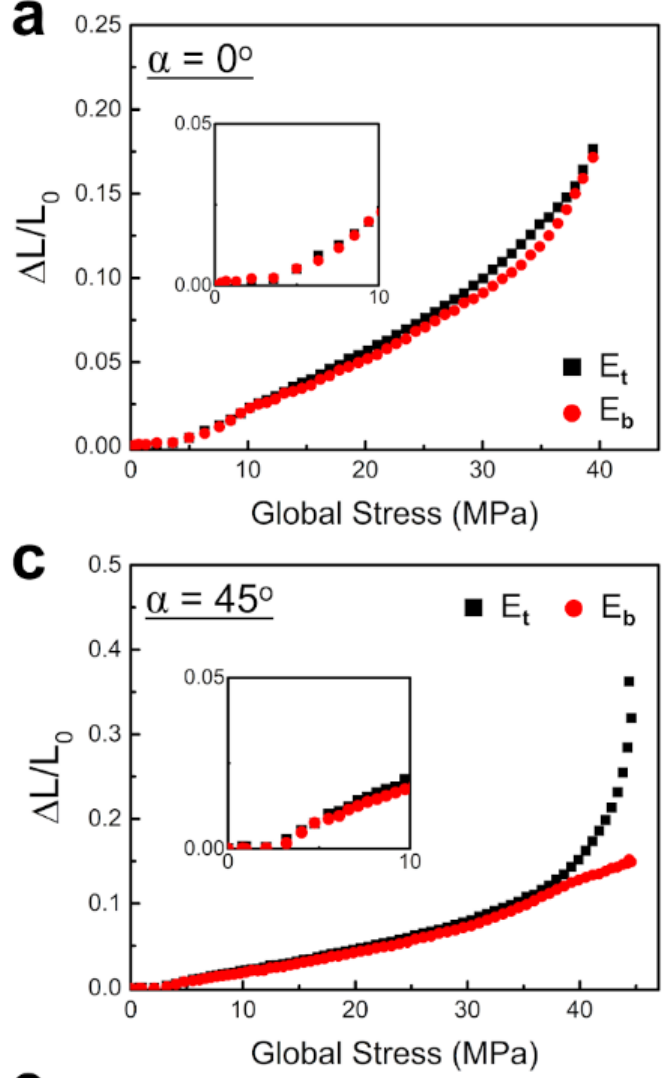

$\mathbf{e}$

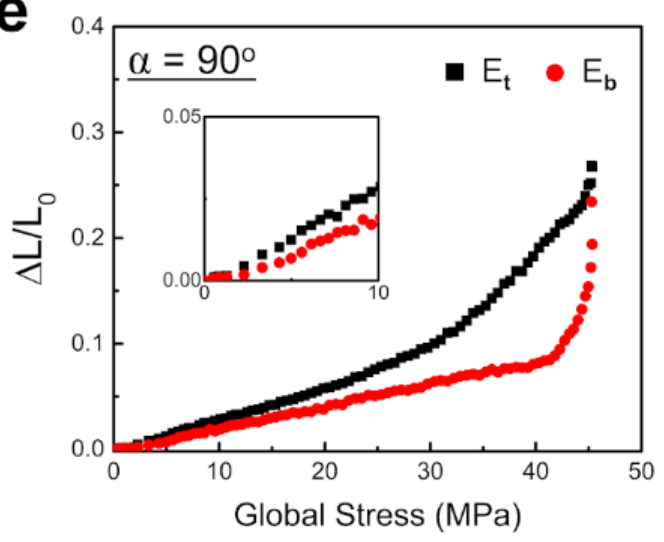


The effects created through the combination of interface debond propagation and nucleation are seen in the matrix failure patterns. Specifically, through the study of the crack propagation angles created in the space between the fibers, we can find critical evidence of the possible reactions of the interaction between fibers due to a remote tensile load. Matrix crack angles measured after postmortem examination of several samples are found in Figure 14. The figure looks at double-fiber samples and compares the respective failure patterns of each angle pair of narrow and wide inter-fiber spacings. The narrow and wide inter-fiber spacing of the figure refers to the least and greatest fiber spacing explored in this work (see Table 2). For all of the narrow inter-fiber cases (marked with a star in Figure 14), it was found that for almost all the samples the measured matrix failure angle was almost identical to the initial angle between the fibers. This behavior lines up with the theory of nearest-neighbor crack linking between the fibers [12-14]. However, this assumption failed to occur in Figure 14d, e where a distinct discrepancy is seen from the pattern seen in the previous samples for the wide inter-fiber spacing. These discrepancies imply a horizontal matrix failure. In the case of these two samples, i.e., samples FF-1.5 $\$-60$ and FF-1.5 $\$-90$, the ultimate failure of the matrix originated from the debonding of the bottom fiber and propagated to the edge of the sample with almost no visible interaction with the top fiber. 


\section{Figure 14}

Matrix failure angle data for double-fiber samples with short (left column) and long (right column) inter-fiber distance. Data shown for samples with (a) $\alpha=0^{\circ}$, (b) $\alpha=30^{\circ}$, (c) $\alpha=45^{\circ}$, (d) $\alpha=60^{\circ}$, and (e) $\alpha=90^{\circ}$. Red circles indicate the original locations of the fibers. Scale bar: $5 \mathrm{~mm}$
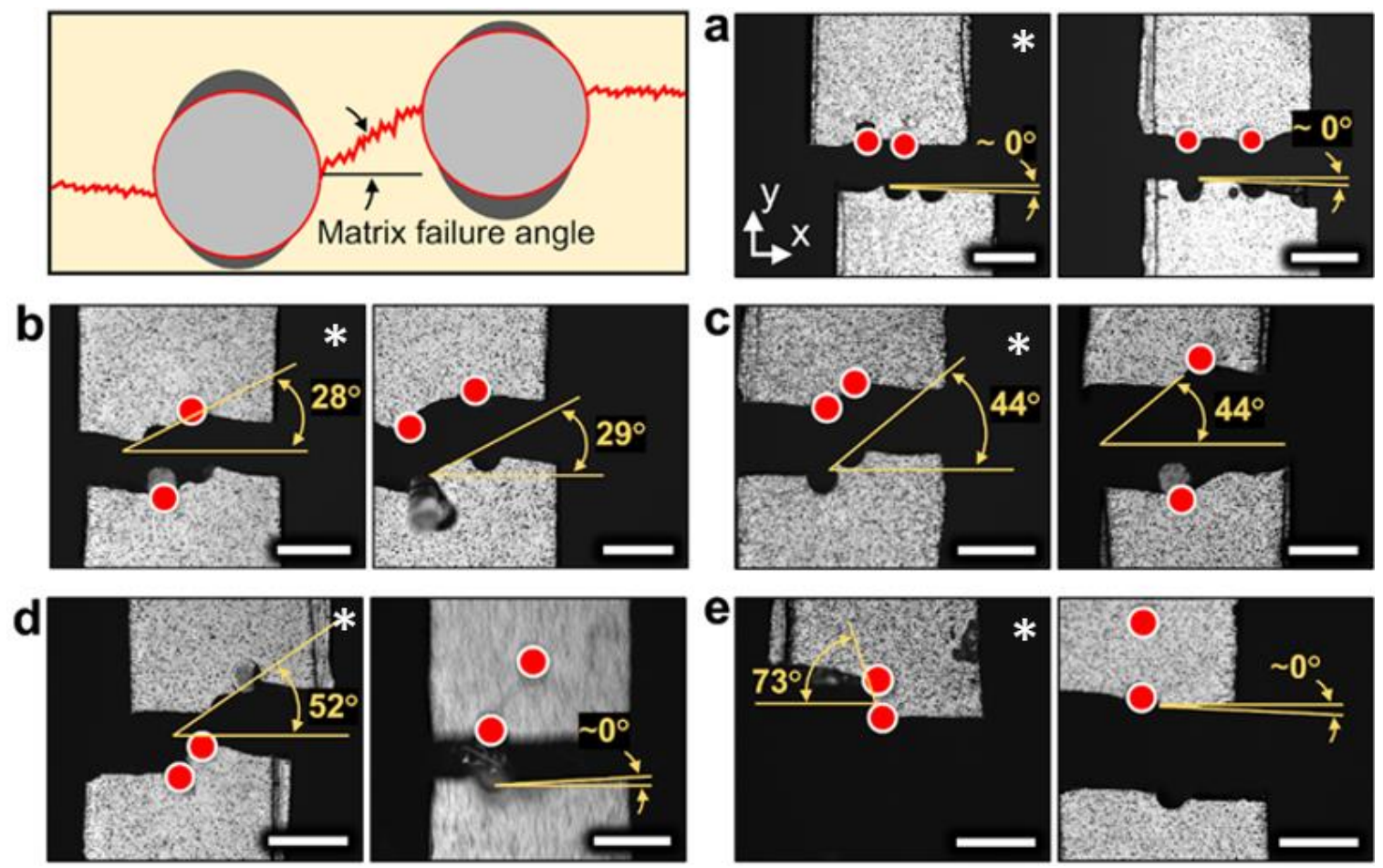

To determine the origin of the previously mentioned abnormalities seen in the samples for $\alpha=60^{\circ}$ and $90^{\circ}$, an examination of the development of shear strain fields between the two fibers was performed. Figure 15 depicts the progression of the local shear strain fields in one of the FF-1 $\phi-30$ samples which feature an initial fiber spacing of ca. $2 \mathrm{~mm}$ and $\alpha=30^{\circ}$. It was found that local shear strain fields developed around the interface at diagonally opposing locations. As stresses rise above $30 \mathrm{Mpa}$, the shear deformation zones tend to develop quicker. These shear bands coalesce together into a single highly deformed strip at >30 MPA. This strip connects the two fibers in the 
shortest distance possible. As displayed in Figure 15b, these highly localized shear bands become the paths along which matrix microcracks originate and follow.

\section{Figure 15}

(a) Evolution of Local Shear Strain Fields in FF-1 $\phi$-30 at Various Global Stresses. (b) A Magnified View of the Epoxy Ligament Between the Two Fibers, Showing the Location of Matrix Crack Initiation

a
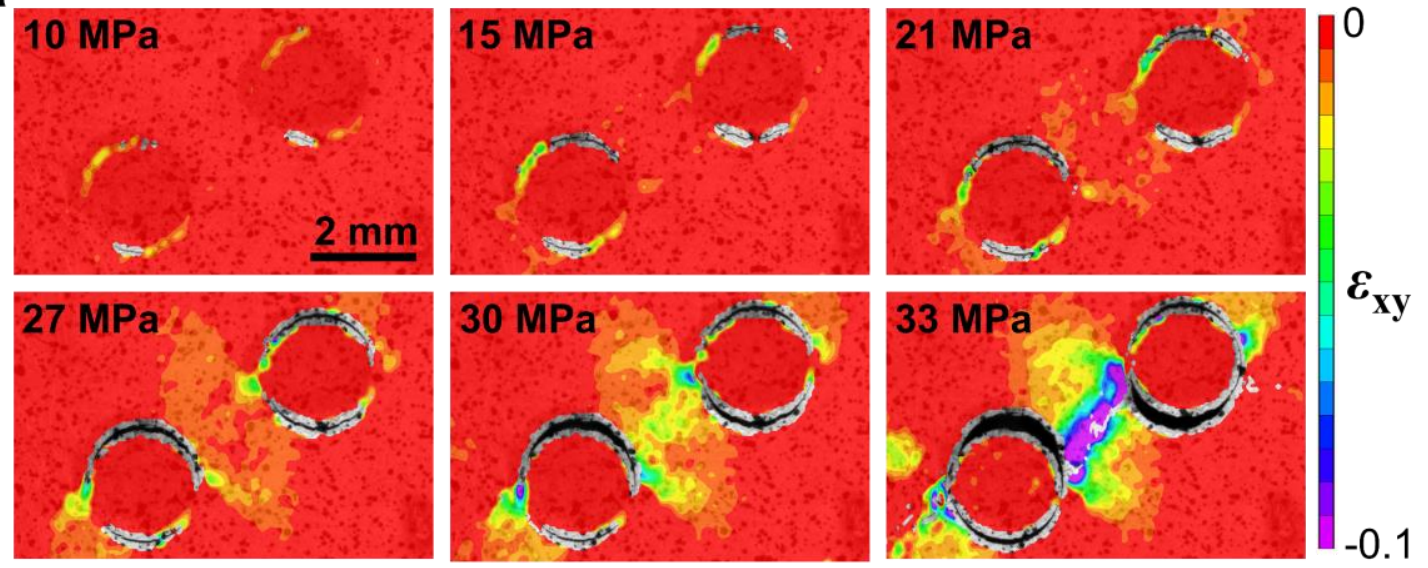

b

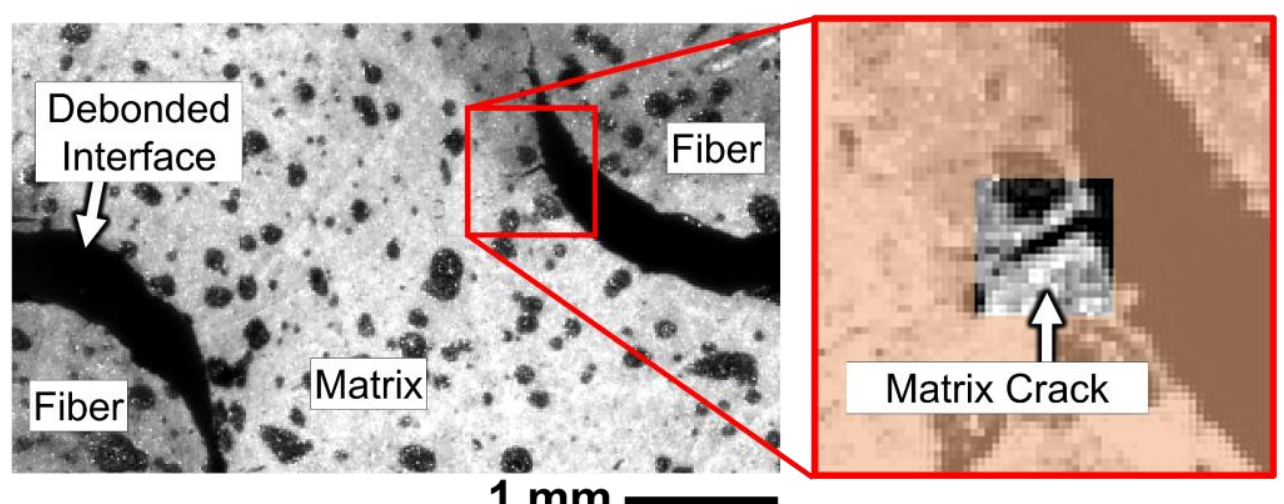

With the previously mentioned observations, the assumption can be made that the coalescence of the shear bands formed between the fibers in multifiber samples (Figure 10) is a primary cause for failure within their matrix. The existence of a link or how 
strong it is between such coalescence of the shear bands with the inter-fiber spacing and angle remains unclear. To address this mystery, a quick study was performed using the assumption of $37^{\circ}$ shear bands (see Figure 10). To begin this study, the normal distance between the inter-fiber spacing and the path taken by the shear bands was established.

Figure 16a depicts a schematic of the geometric features utilized in this study. The color map seen in Figure 16b illustrates the correlation for each sample based on its inter-fiber distance and its shear band distance, $\mathrm{L}$. The negative values seen on the color map indicate the instances where the inter-fiber angle is less than the shear band angle, i.e., $\alpha<37^{\circ}$. The next step is through matrix failure measurements taken postmortem (like in Figure 14), samples were separated based on whether their shear bands coalesced or if the matrix failed without any apparent interaction between fibers occurring. This separation is illustrated by marking the $(\alpha, d)$ pairs on Figure 16b with either a solid or a hollow circle based on their respective failure property. Surprisingly, all samples with $d / \phi$ ratios $<0.5$ regardless of inter-fiber angle demonstrated matrix crack coalescence. As $d / \phi$ ratios increase above 0.5 , the inter-fiber angle appears to play a major role in the probability of the occurrence of shear band coalescence. Based on the data acquired, an apparent division between whether coalescence will occur or not seems to have formed where $d / \phi>1$ and $\alpha>45^{\circ}$. While the reasoning for this is not investigated in this work, we theorize that this phenomenon may directly be in relation to interactions existing between the plastic zones and the stress concentration fields that exist between the neighboring cracks $[31,32]$. Even so, based on the experimental findings of this work it may not be realistic to assume coalescence between neighbor fibers and crack linking. In light of this, more accurate predictions for transverse cracking and ultimate matrix failure in 
unidirectional composites will necessitate more comprehensive information concerning the inter-fiber distance and angle between the nearest neighbors. This is especially important for the study of composites containing dilute fiber packings [33].

\section{Figure 16}

(a) Schematic of the Geometric Features of the Shear Band Coalescence Analysis, Sowing the Normal Distance Between the Trajectory of the Shear Bands, Denoted by L. (b) The Correlations Between L, A, and D/ $\phi$ Ratio Shown as by the Color Map. The (A, $D / \phi)$ Pairs Wherein the Coalescence of Shear Bands Were Observed (or Not Observed) Are Shown by the Overlayed Black or Hollow Circles
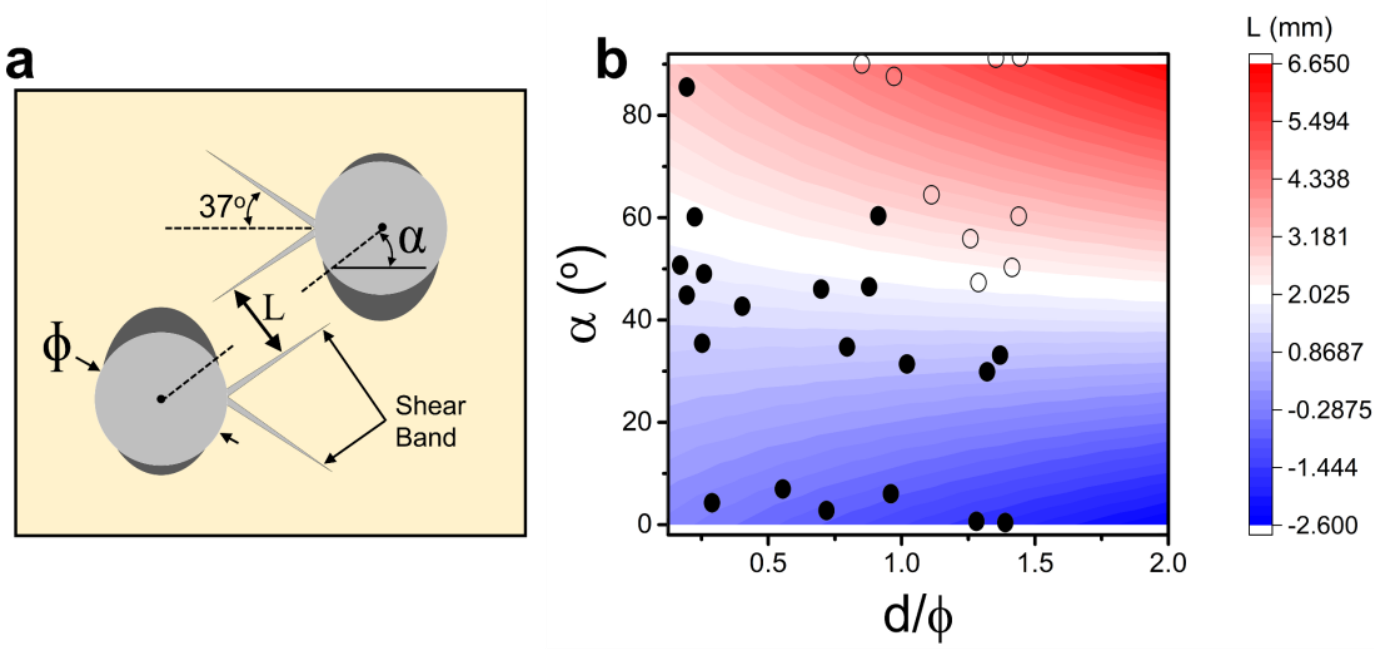

Shear band coalescence observed

Shear band coalescence not observed

\subsection{Tensile Strength Measurements}

When testing, the tensile strengths of the double-fiber samples were drawn with the trends seen previously (see Figure 13) concerning the interface opening response. Figure 17 displays how the ultimate tensile strength (UTS) of the samples varied based on their respective inter-fiber angles and distances with lines for the neat and single fiber 
UTS included for comparison purposes. This figure is comprised of data from all of the double-fiber samples tested, as such, it is capable of providing data about the variability on a sample-to-sample basis. Despite not suggesting any specific failure mechanisms, such as interface debond initiation or propagation, the results displayed in Figure 17 do provide a look into stress concentration and matrix failure allowing for a deeper understanding of possible fiber interaction mechanisms. The patterns seen in the tensile strength data for the samples mimic those of the classical multiple crack interactions problem solution [34]. When working with coplanar fibers, i.e., $\alpha=0^{\circ}$, it has been found that by reducing the size of the bridge connecting the two fibers, the region over which the far-field tensile load can be conveyed is also reduced. As a result, the stress concentration is amplified. This amplification allows matrix failure to occur at far lower global stresses. As the inter-fiber distance increases, the overall effect the stress concentration factor has on the sample weakens, thus allowing the sample to endure increased forces before fracture and ultimately failure. When this occurs the double fiber sample behaviors will mirror that of a single fiber sample due to the effect the neighboring fiber usually has on the ultimate tensile strength of the sample being heavily diminished. The double-fiber samples acting like single fiber samples are shown to be exhibited as the inter-fiber spacings exceed that of the fiber diameter which in this case is $2 \mathrm{~mm}$. 


\section{Figure 17}

Variation of Tensile Strength for Different Inter-Fiber Distance and Angle Conditions. Tensile Strength of Neat Epoxy and Single-Fiber Samples Are Shown for Comparison

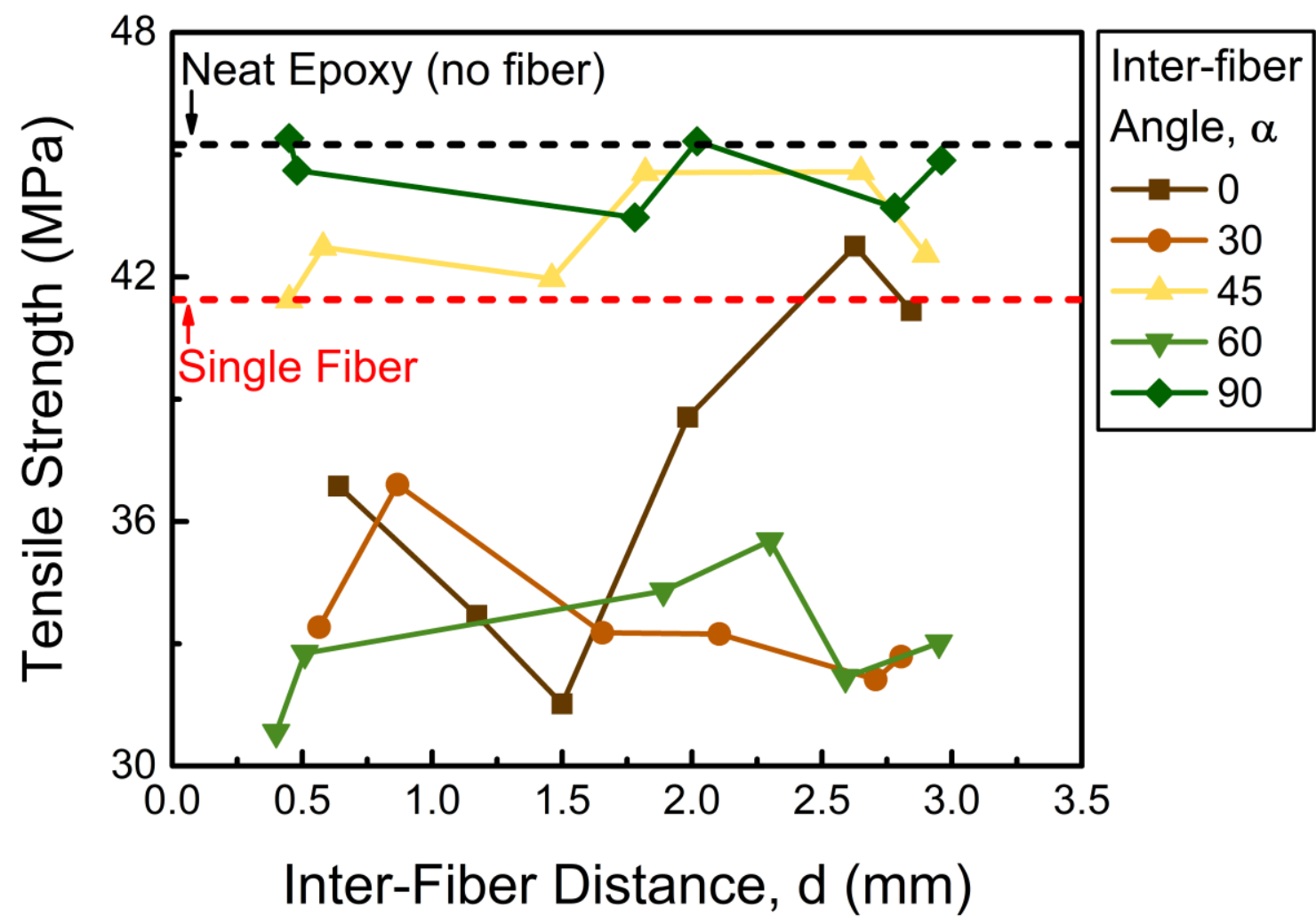

When samples with their fibers aligned parallel to the tensile load direction, i.e., $\alpha=90^{\circ}$, were tested a completely unique behavior was observed. Once again mimicking the parallel crack conditions found in classical fracture mechanics [34], there is clear evidence of the existence of a shielding mechanism. In the case of the samples tested, this shielding mechanism means that the two fibers and their respective debonded interfaces will tend to protect each other. Through this shielding phenomenon, the single fiber case experiences relatively less overall stress concentration, and in the case of the double-fiber samples the shielding allows for enhanced resistance to failure. Conversely in the case of 
parallel cracks, as the ligament connecting the two cracks grows in size it has the potential to increase the overall stress concentration and thus drastically increase the sample's susceptibility to premature fracture and failure. While the double-fiber's behavior is not evident in this work, the overall tendencies suggest the double-fiber samples with $\alpha=90^{\circ}$ have a greater UTS.

Lastly, when the behaviors for $\alpha=30^{\circ}, 45^{\circ}$, and $60^{\circ}$ are observed a surprisingly counter-intuitive effect emerges. When testing it was found that the failure strength of the $\alpha=45^{\circ}$ sample when measured was significantly greater than that of either the $\alpha=30^{\circ}$ or $60^{\circ}$ sample. The reason for this behavior is possibly in relation to mixed-mode stress concentrations developing in the sample [34]. Unfortunately, this assumption currently has no concrete experimental data available to support it. Nonetheless, this work seeks to further understanding of the fiber interaction mechanisms by studying the shear band coalescence response of fibers. 


\section{Chapter 5}

\section{Summary}

The impact of the inter-fiber spacing and angle on the interaction between two neighboring fibers was determined through experimental measurements. Samples of both the single and double-fiber variety were fabricated by embedding glass macro fibers in specified orientations in an epoxy matrix. High magnification optical digital image correlation was utilized for characterizing the strain fields surrounding the fibers. Initially, the global and local deformation responses in single fiber samples were determined. These results were used for the calibration of the finite element model in addition to illustrating the deformation patterns within the area surrounding a fiber that is under transverse tension. The data obtained from single fiber samples were also used to calibrate a simple bi-linear traction-separation law that was used in FE models. This understating was then used to examine double-fiber samples and their associated mechanisms for fiber-matrix debonding and matrix failure. It was shown that the interfiber matrix failure in tightly packed composites is affected by the coalescence of shear bands originating from the fiber-matrix interface in neighboring fibers. It was also found that the coalescence of the shear bands is, to some extent, dependent on the inter-fiber spacing and orientation between neighboring fibers. Lastly, it was found that inter-fiber matrix cracking and coalescence of the shear bands have a lower chance of occurring when the nearest fiber is positioned further than a single fiber diameter away and is also positioned at an angle less than $45^{\circ}$ in relation to the tensile load direction. 


\section{Chapter 6}

\section{Recommendations}

While this research provided a detailed insight into the mechanics of failure associated with fiber-matrix debond, there are still future works that can be done to further refine these results and deepen our understanding of the underlying mechanics of fiber-matrix debonding and matrix failure phenomena. The following are a list of recommendations provided for the expansion and further improvement of this research:

- First, a better jig and casting mold are to be designed to more easily maintain proper placement of fiber spacing and angle during sample fabrication to ensure consistent sample preparation.

- Increase the number of fibers to explore the effects that fiber bundles create when several fibers are in close proximity to see if an increase in the number of fibers magnifies the rate of the debond and the rate at which propagation of the debond occurs.

- Explore the effects of thermal modulation. This is especially imperative in the case of aerospace composites where components experience large changes in thermal conditions.

- Use 3D DIC in one of two ways

- Use the two cameras for traditional 3D (stereo) DIC to allow for the measurement of out-of-plane deformation and strain fields

- Take advantage of the transparency of the epoxy sample and set up the cameras orthogonal to each other and perpendicular to the front and side 
faces of the sample. Doing this will allow one camera to track the front face of the sample and thus gather strain data while the second camera tracks the fiber through the transparent epoxy sample to study the internal debonding patterns. The latter would be of significant interest in characterizing post-debond behavior and crack tunneling along fibers. 


\section{References}

[1] Martyniuk K., Sorensen B.F., Modregger P., Lauridsen E.M., 3D in situ observations of glass fiber/matrix interfacial debonding. Composites Part A 2013; 55: 63-73.

[2] Talreja R., Transverse cracking and stiffness reduction in composite laminates. Journal of Composite Materials 1985; 19: 355-375.

[3] Zhuang L., Talreja R., Varna J., Transverse crack formation in unidirectional composites by linking of fibre/matrix debond cracks. Composites Part A 2018; 107: 294303.

[4] Montgomery C.B., Koohbor B., Sottos N.R., A robust patterning technique for electron microscopy-based digital image correlation at sub-micron resolutions. Experimental Mechanics 2019; 59: 1063-1073.

[5] Paris F., Correa E., Mantic V., Kinking of transversal interface cracks between fiber and matrix. Journal of Applied Mechanics 2007; 74: 703-716.

[6] Tan W., Naya F., Yang L., Chang T., Falzon B.G., Zhan L., Molina-Aldareguia J.M., Gonzalez C., Llorca J., The role of interfacial properties on the intralaminar and interlaminar damage behaviour of unidirectional composite laminates: Experimental characterization and multiscale modelling. Composites Part B 2018; 138: 206-221.

[7] Velasco M.L., Graciani E., Tavara L., Correa E., Paris F., BEM multiscale modelling involving micromechanical damage in fibrous composites. Engineering Analysis with Boundary Elements 2018; 93: 1-9.

[8] Velasco M.L., Correa E., Paris F., Interaction between fibers in the transverse damage in composites. Engineering Fracture Mechanics 2020; 239: 107273.

[9] Shakiba M., Brandyberry D.R., Zacek S., Geubelle P.H., Transverse failure of carbon fiber composites: Analytical sensitivity to the distribution of fiber/matrix interface properties. 2019; 120: 650-665.

[10] Zhang X., Brandyberry D.R., Geubelle P.H., IGFEM-based shape sensitivity analysis of the transverse failure of a composite laminate. Computational Mechanics 2019; 64: 1455-1472.

[11] Garcia I.G., Mantic V., Graciani E., Debonding at the fibre-matrix interface under remote transverse tension. One debond or two symmetric debonds? European Journal of Mechanics A/Solids 2015; 53: 75-88.

[12] Vejen N., Pyrz R., Transverse crack growth in glass/epoxy composites with exactly positioned long fibres. Part I: experimental. Composites Part B 2001; 32(7): 557-564. 
[13] Zhuang L., Pupurs A., Varna J., Talreja R., Ayadi Z., Effects of inter-fiber spacing on fiber-matrix debond crack growth in unidirectional composites under transverse loading. Composites Part A 2018; 109: 463-471.

[14] Sandino C., Correa E., Paris F., Numerical analysis of the influence of a nearby fibre on the interface crack growth in composites under transverse tensile load. Engineering Fracture Mechanics 2016; 168: 58-75.

[15] Zhang W., de A. Melo L.G., Hitchcock A.P., Bassim N., Electron beam damage of epoxy resin films studied by scanning transmission X-ray spectromicroscopy. Micron 2019; 120: 74-79.

[16] Rashidi A., Olfatbakhsh T., Crawford B., Milani A.S., A review of current challenges and case study toward optimizing micro-computed X-ray tomography of carbon fabric composites. Materials 2020; 13: 3606.

[17] Tracy J., Daly S., Sevener K., Multiscale damage characterization in continuous fiber ceramic matrix composites using digital image correlation. Journal of Materials Science 2015; 50: 5289-5299.

[18] Koohbor B., Ravindran S., Kidane A., A multiscale experimental approach for correlating global and local deformation response in woven composites. Composite Structures 2018; 194: 328-334.

[19] Koohbor B., Ravindran S., Kidane A., Meso-scale strain localization and failure response of an orthotropic woven glass-fiber reinforced composite. Composites Part B 2015; 78: 308-318.

[20] Mehdikhani M., Aravand M., Sabuncuoglu B., Callens M.G., Lomov S.V., Gorbatikh L., Full-field strain measurements at the micro-scale in fiber-reinforced composites using digital image correlation. Composite Structures 2016; 140: 192-201.

[21] Koohbor B., Montgomery C.B., White S.R., Sottos N.R., Meso-scale strain measurements in fiber reinforced composites. 33rd Technical Conference of the American Society for Composites 2018; Seattle, WA, USA. DOI 10.12783/asc33/26028 (http://www.dpi-proceedings.com/index.php/asc33/article/view/26028)

[22] Tabiai I., Delorme R., Therriault D., Levesque M., In-situ full field measurements during inter-facial debonding in single fiber composites under transverse load. Experimental Mechanics 2018; 58: 1451-1467.

[23] Tabiai I., Texier D., Bocher P., Therriault D., Levesque M., In-situ full field out of plane displacement and strain measurements at the micro-scale in single reinforcement composites under transverse load. Experimental Mechanics 2020; 60: 359-377. 
[24] Tabiai I., Tkachev G., Diehl P., Frey S., Ertl T., Therriault D., Levesque M., Hybrid image processing approach for autonomous crack area detection and tracking using local digital image correlation results applied to single-fiber interfacial debonding. Engineering Fracture Mechanics 2019; 216: 106485.

[25] Vaughan T.J., McCarthy C.T., Micromechanical modelling of the transverse damage behaviour in fibre reinforced composites. Composites Science and Technology 2011; 71(3): 388-396.

[26] Rajan S., Sutton M.A., Fuerte R., Kidane A., Traction-separation relationship for polymer-modified bitumen under Mode I loading: Double cantilever beam experiment with stereo digital image correlation. Engineering Fracture Mechanics 2018; 187: 404421.

[27] García I.G., Mantič V., Graciani E., Debonding at the fibre-matrix interface under remote transverse tension. One debond or two symmetric debonds? European Journal of Mechanics A/Solids 2015; 53: 75-88.

[27] Goodier J., Concentration of stress around spherical and cylindrical inclusions and flaws. Journal of Applied Mechanics 1933; 55: 39-44.

[28] Mantič V., Interface crack onset at a circular cylindrical inclusion under a remote transverse tension. Application of a coupled stress and energy criterion. International Journal of Solids and Structures 2009; 46(6): 1287-1304.

[29] Dundurs J., Discussion of a paper by D.B. Bogy. Journal of Applied Mechanics 1969; 36: 650-652.

[30] Song S.H., Choi B.H., Effect of plastic zone on the fatigue crack propagation behavior between two fatigue cracks. Experimental Mechanics 2001; 41: 225-231.

[31] Koivisto J., Dalbe M.J., Alava M.J., Santucci S., Path (un)predictability of two interacting cracks in polycarbonate sheets using digital image correlation. Scientific Reports 2016; 6: 322278.

[32] Anderson T.L., Fracture Mechanics - Fundamental and Applications, $3^{\text {rd }}$ editions. Taylor \& Francis, 2005. 